\title{
VIERTE ABTHEILUNG.
}

\section{Botanische und pharmakognostische Neuig- keiten; mitgetheilt von Dierbach.}

1. Schrifteil und Abbildungen zur Kenntnifs der Arzneipflanzen und der vegetabilischen Droguen.

Um die schon seit einer Reihe von Jabren beobachtete Anordanng dieses Berichtes nicht 20 verändern, beginnen wir wieder mit einer Uebersicht der neuesten Leistungen im Gebiete der medicinisch.pharmaceutischen Botanil and Iconographie. Es gehört hierher zurơrderst:

Abbildure und Beschreibung aller in der Pharmacopoea borussice aufgeführten Gewächse. Herausgegeben ron Friedrich Guimpel, Prof. bei der königl. Academie der Künste za Berlin, Text von D. F. L. V. 8chlechtendal, Prof. der Botanik in Halle.

Wie bei den früheren Berichten müssen wir zurördernt wieder auf deo später gelieferten Text zo den bereits im vorigen Jahro gelieferten Abbildungen zurüchknmmen, um auch jetzt wieder das Bedauern zn äufsern, dafs dioser to äulserst langsam und spät geliefert wird, wie denn sogar derselhe noch nicht einmal die Erblärung der bereits 1835 gelieferten Kupfer vollatändig entbält. Bei Quassia amara bemerkt Herr v. Schlechtendal: das im Handel verläufliche zerschnittene Quassienholz zeige oft Spuren von Fernambuls uder Sappanholz, welches bei nicht gehöriger Reinigung der Nühien darunter gerathe; das Decoct eines solchen verunreirigten Holzes werde von schwefelsaurem Eisenoxydul getrübt, währead das des reinen Quassienholzes ungetrübt bleibe 
Als Mutterpflanze der Ruhrrinde ist blos Simaruba officinalis (Quassia Simaruba Wright) abgebildet und beschrieben; sebr gerne würde man aber auch die verwandte Simarnba amara Hayne gesehen haben, deren Rinde, wie auch der Herr Verf. bemerht, ganz anfserordentlich bitter ist. Nur bis zn No. 260 reicht der Text and begreift hanptsächlich die cultivirten Arten ron Triticam, über die aber nichts Neues oder Eigenes mitgetheilt wird.

Band III. Heft 11 and 12.

No. 261. Croton Tiglium.

- 263. Vanilla aromatica.

-263. Laurus Camphora.

- 364. Lauras Cassia.

- 265. Laurus Cinnamomum.

2 266. Laurus nobilis.

267. Laurns Sassafras.

" 968. Dracaena Draco.

269. Chenopodium ambrosioides.

* 270. Chenopodiam Botry8.

7 271. Pistacia Lentiecus.

\279. Pistacia Terebinthos.

Band III. Heft 13 und 14 .

No. 273. Alpinia Cardamomum.

274. Illicium anisatum.

- 275. Cucumis Colocynthis.

- 276. Ficus religiosa.

- 277. Ficus indica.

- 278. Myroxylon peruiferum.

D 279. Myroxylon toluiferum. (Blos ein gefiedertes Blatt)

"280. Balcamodendron Myrrha.

ข 281. Balsamodendron Kataf. 
No. 282. Geoffroya surinamensis.

\283. 284. Brassica Rapa mit Varietäten.

Noch wenige Hefte sind jetzt nur noch erforderlich, um alle PAlanzen in Abbildungen za lieforn, die nach der Pharmacopoea borassica officinell za bewahren sind; es soll nan aber noch ein Supplement erscheinen, in welchem Herr Prof. Guimpel die Abbildangen jener Gewächse zu geben beab. sicbtigt, welche theils in andern Pharmacopøen, theils in den Schriften berühmter älterer Aerzte aufgetührt aind, anch davon wird das Heft za dem in der That billigen SubscriptionsPreise ron $15 \mathrm{Sgr}$. erlassen, und man darf um so mehr etwas Yorzügliches erwarten, da dem Herm Heransgeber nicbt blos der $s 0$ reiche bönigl. botan. Garten in Berlin, sondern auch die dortige Bibliothek und die kŏnigl. Herbarien zu benutzen gestattet ist.

Merkantilische Warenkunde, oder Naturgeschichte der vorzũglichsten Handelsartilel mit illuminirten Abbildungen, ron Dr. Ernat Schenk. Ein unentbehrliches Hülfsmittel für Kaufmünner, Drognisten, Färber, Technologen und alle diejenigen, welche eine gründliche Warenlenntnifs zu erlangen beabsichtigen. Nach den besten Quellen bearbeitet ron Jonathan Carl Zenker, Dr. der Philos., Medicin und Cbirurgie, ordentl. Prof. dor Naturgeachichte und Botanil an der Unirersităt Jena. Band 3. Heft 1. Jena 1835 , bei $A$ ugust Schmid.

Ueber den Inhalt der beiden ersten Bände dieses auggeseichnet schönen und nützlichen Workes ist bereits früher (Annalen der Pharm. Bd. 14. S. 237.) Bericht erstattet wordeb. Das rorliegende Heft enthält folgende Abbildungen, die man unbedingt zu den gelungensten and instructivaten zählen kann, welche die medicinisch-pharmacentische Botanils oufzuweisen hat. 
Tab. 49. Quercus Aegilops L. Hnopper-Eiche.

Quercus infectoria Olliv. Galläpfel-Eiche.

\50. Quercus Saber L. Kork-Eiche.

D 51. Caecalpinia echinata L. Stachlige Cäsalpinie.

》52. Cacealpinia Sappan L. Sappan ,Cäsalpinie.

- 53. Carthamus tinctorius L. Färbe-Saflor.

\54. Boletus igniarius L. Feuerschwamm.

Boletes fomentarius $\boldsymbol{L}_{\boldsymbol{w}}$ Zunderschwamm.

Boletus marginatus Persoon. Fichtenschwamm.

Es ist dem Ref. lein Werh belsannt, in welchem dio Naturgeschichto and überhanpt alles Wiesenswerthe der angezeigten südlichen Eichen so umfassend, gründlich und belehrend onseinandergesotzt wäre, als in dem rorliegenden, und diese allgemeine Bemertung kann man mit Recht auch onf die übrigen genannten Gewüchse nusdehnen; mit ganz besonderem Fleifse und rühmlicher Belesenheit sind insbecondere die Caesalpinien abgebendelt, und mit Vergnügen wird man die Nachrichten von den verschiedenen Arten der Gattong Boletus, die zur Bereitung des Zunders dienen, nachlesen.

Getreue Darstellung und Beschreibung der in der Arzneibunde gebräuchlichen Gewächse, wie auch solcher, welche mit ihnen verwechselt werden tönnen. Von Dr. Friedrich Gottlob Hayne, ordentl. Professor an der Lơnigl. Friedrich-Wilhelms-Universität zu Berlin. XII Bandes I-IV Lieferang. Berlin. Aaf fosten des Ver. fassere.

Mit Vorgnügon zeigt Bef. das Dasein mehrerer noven Lie. ferungen dieses Werkes an, das so wesentlichen Einflufe auf die genauere und richtigere Kenntnifs der Arzneipflanzen

-) Die Namen der jetzigen Herrc: Herauggeber dieses Werkes sind auf dem Umachlage der voriegenden Hefte nicht genannt. 
batte, und immerhin als oin Muster ron Grindliahleit und deatsehem Fleifse anerhannt werden wird. Man findet hier folgende Abbildungen:

Tab. 1. Rumex obtusifolius. Fig. sinistra

Rumex sylvestris. Fig. dextra.

》2. Rumex Oxylapathum.

b. Bumex crispus.

" 4. Rumex aquaticus. Fig. sinistre. Rumex Hydrolapathem. Fig. dextra.

Die Ampfer-Arten, ron denen die Radix Lapathi acuti der Officiben gesammelt werden soll, so wie die geasue Bestimmung des wabren Romex acutus Lin naei beschäftigte schon viele Botaniker, und die genaue Onterscheidang dieser so gemeinen und weit verbreiteten Pflanzon ist in der That richt ganz leicht. Mit grofsem Fleifse wird man bier diese verwickelte Materie bearbeitet finden, and die herrlichen $\mathbf{A b}$ bildnngen werden zur richtigen Beartbeilung des Ganzen wesentlich beitragen. Möge man nun auch nicht alle hier abgebildeten Formen für wahre Arten anerkennen, oder soch in Hinsicht der Synonymie da und dort abweicheade Ansichten haben, so sind doch hier die erforderlichen Materialien mit solcher Klarheit und Deutlichbeit angeordnet, dafs man dieser Arboit nur mit grolsem Lobe gedenten hann.

Taks, 5. Rumex Dioscoridis: foliis oblongo-lanceolatis acutis, radicalibus basi coarctatis, caulinis inferioribus dilaiatis, valpulis ovato-rotundis, basi cordatis integris vel den. ticulatis, nnica tantum callo minuto oblongo acumioato minuto.

Es ist diels eine neu aufgestellte Art, die man aus Saamen 208, wolche mit dem Opium aus dem Orient kamen, das bekanntlich in Ampferblätter eingewichelt rerschicht 20 werden pflegt. Wall roth nannte die Pflanze zuerst Mumex macrophyllus, änderte aber später diesen Namen in Rumex Dioscoridis ab, indem er glaubte das Oxỵlapathon dieses grie- 
chischen Pharmakologen in der hier in Redo atehenden Planza gefunden zu baben. Sie ist dem englischen Spinat oder Rumex Patientia sebr ähnlich und hauptsächlich nur darch die Form der Valvalae unterschieden. Uebrigens wird das Opinm in die Blätter mehrerer Arten von Humex eingevickelt; 80 erzog Herr Apothelser Biltz den Rumex crispus aus in Opium gefundenen Samen, und Bernhardi erzog aus solchen gowôbnlich Rumez Patientia, oder oine Varietät derselben, die er A. Patientia $\beta$ orientalis nannte.

Tab. 6. Rumex Acetosa, deren Varietäten mit groluex Sorgfalt auseinandergesetzt sind. Zn der Waldform R. Ace tosa silvaticus wird Rumex arifolius Allioni and R. hiapanicus Gmelin Flor. Bad. gezogen.

Tab. 7. Rumax alpinus. Die Erinnerung an diese alte Arzneipflanze ist gewift an ihrem Orte, indem die bior nicht erwähate Entdeckung Geiger's (Rumicin) eine ungemein grofse Verwandtschaft mit der Wirkang der Rhabarbor exwarten läfst. Die Erörterung, was die Alten unter dem Namen Rheum Monachorum verstanden, wie sie sie benutzten, und welche Wirkang sie davon sehen, dürfte jetzt wieder von besonderm Interesse seyn, wenn man damit die neueren chemischen Ansichten und Erfahrungen verbindet.

Tab. 8. Cyclamen europaeum.

- 9. Lobelia antisyphilitica (ganz passend statt syphilitica genannt).

Tab. 10. Fraxinus excelsior. Mit sehr genauer und ausführlicher Frơrterung der zablreichen Varietäten dieses deotschen Waldbaumes.

Tab. 11. Ornus europaea.

- 12. Ornus rotundifolia.

Ganz vortreffliche Abbildungen, selbst die Frucht der MannaEsche (aus der Flora graeca copirt), nebst den zaverlässigen botaniscien Bestimmungen von Tenore in Neapel. 


\section{Botanische und pharmakognostische Newigkeiten.}

Tab. 13. Pyrola nmbellata.

Man vermift hier ungera die $\Delta$ ngabe der speciellen chemischen Analyse des doldenförmigen Wintergrŭns, welehe Apatheser Martens in Leipzig lieferte.

Tab. 14. Chenopodium Botry8.

15. Chenopodiam ambrosioides.

x. Morus nigra.

17. Juglans regia.

ग 18. Pistacia rera.

7 19. Pistacia Terebinthus.

20. Pistacia Lentiscus.

Uober die eigenthümlichen Auswüchso des Terpentinbaums (Gallae Terebinthi), die jetzt anch in Deutschland als Arzneimittel benutzt werden, würde man sehr gerne spociello Angaben und eine Aobildung geseben habea.

Tab. 21. Polygala Senega. Schöno Abbildung des ganzen blühenden Gewāchses, nebst einem frachttragenden $\mathrm{Z}$ weige nach Exemplaren, die Herr Kaufwann Preskatt in Peterz. burg mittheilte, nebst genauer Analyse und interessanten Bemerhongen im Texte.

Tab. 22. Polygala amara. Fig. l. P. austriaca Cranz Fig. IL. P. uliginosa R eicben bach. Fig III. P. amarella Granz.

Tab 23. Polygala volgaris Fig. I varietas major. Fig. II. varietas oxyptera collina. kig. III. varietas oxyptera pratensis. Fig. IV. V. Spielarten dieser letzteren nach den Blumenfarben.

Tab. 24. Polygala vulgaris. Fig. I. rar, amblyptera glabra. Fig. II. var. amblyptera pubescens. Fig. IIL. Polygala alpestris. Fig. IY. P. serpyllacea.

Zu wünschen wären noch Polygala major Jacqua und $P$. comosa Schkuhr, so wie specielle Angaben über die Stand- 
Botanische und pharmakognostische Neuighetten. 249

Orter, wo die P. amara für die Officinen einzuaammeln ist, worüber Ref. anderwärts ausführlich seine Erfabrungen mittheilte.

II. Nene Nachrichten über griechische Arzneipflanzen.

Eo ist eine allbehannte Thatsache, dafs unsere heutige Materia medica, so verschieden sie auch von der des Alterthums seyn mag, dennoch aus dieser herworging, die ihr als Basis diente. Der groffte and beste Theil der noch heat 24 Tage gebräuchlichen Arzneiplanzen war schon den alten griechiscben Aerzten belannt, and was sie von ibren Wire knagen sagen, beatätigto grofsentheils dio Erfabrung vieler Jahrhunderte. - Jeder Beitrag zar näheren Benntnifs diesor primitiren Heilmittel mufs daher ron hohem Interosse sojn, ond es wäre tadelnswerth, wonn in diesen Debersichten das, was hierüber die neueste Zeit lehrte, übergangen werden sollte. Wenn in dem vorigen Jahrhunderte noch es vorzugsweise Britten waren, die Griochenlande botanische Schätze zu erforechen, uad die alten Heilpflanzen $z 0$ ermitteln suchren, so haben in den nouesten Zeiten Franzosen und Deutsche die günstigo Gelegesheit nicht rersäumt, ein Gleiches za thou. Niomand wird es verargen, wenn hier zuerst von dea Bemühnagen der Letzteren die Rede ist.

H8chst schïtabare Nachrichten verdenken wir zurơrderst dem Herrn Professor Linh in Berlin, welcher im Sommer 1833, in Gesellschaft des berübmten Geognosten ro n Buch, einen Theil jener asch für Botanik and Materia medica Hlassischen Länder bereiste, die Flor der schönen Insel Zante (Zacynthus) begrülste, die hohen Gebirge um Nauplia bis nach Argos hin durcheuchte, nicht minder die Gegend um $\Delta$ then, in Gesellschaft des Pharmaceuten Sartorius den im 
Alterthum so berühmten Berg Hymettus bestieg, dio Gegend ron Korinth, die 80 viele Erinnerungen herrorrufende Ebne ron Eleasis sah u. s. W. Eine Uebersicht der in Griechenland gesammelten Pllanzen theilte Hr. Prof. L. in der Linnaca des Hrn. v. Schlechtendal mit, die hier benutzt ist. Daran schliefsen sich die Nachrichten des Apothekers Landerer in Athen, welche in Buchner's Repertorinm mitgetheilt wurden.

Plantago Coronopus ist nach Landerer ein griechiacbes Hausmittel, das im Decocte als Diureticum bei der Hanch wassersuoht benztzt wird. Man wendet dazu die ganze Planze an, wenn sie darch die Sonnenhitze schon ganz ansgetrocknet ist. - Nach Link kommen in den Seegegenden Griochenlands, namentlich um Patras, mebrere Arten ron Plantago ror, die unter dem Namen P. Coronopus theils ron Te. nore, theils ron französisohen Botanikern beschrieben wrurden, und die als Arten zu unterscheiden sind, wie Plantago commutata Gussone, P. neglecte Gussone, woza Hr. Prof. Lial P. ceratophylla seiner Flora ron Portugal rechnet; übrigens darf man wohl annehmen, dals in medicinischer Hinsicht diese Unterschiede ron geringer Bedentung sind. Schon Dioscorides erwähat Plantago Coronopus als Ara neimittel, das er zumal bei gewissen Unterleibskrankheiteo anräth; allein eine grofse Wirhsamkeit wird man daron nicht erwarten dürfen, da in jenen Zeiten die Pflanze da Gemiese diente und mithin sich ziemlich indifferent rerbält.

Cyclamen graecum Link foliis hysteranthiis subrotundis crenulatis subtus raberrimis, corollae fauco dentata. Dieso neue Art fand/Hr. Prof. L. häufig an Felsen um Nauplie, im September blühend. Die Arten von Cyclamen gehören zu den ältesten Arzneimitteln, und es darf daher auch diese neue Species nicht übersehen werden.

Origanum smyrnacum $L$. fand Hr. Prof. L. hëafig on 
Felsen in Griechealand and erinnert dabei, dafs die Herbe Origani cretici der Otficinen rorzugerroise ron dieser Species genommen werdo.

Menthe niliaca Jacquin fand Hr. Prof. Lo bänfig an feuchten Orten am Nauplia und anderwärts in Griechenland; er sotzt hinzo: die Planze lat sicher von Mentha silvestris verschieden, die Blätter sind schmüler, die Quirlen fast alle getrennt and eine lange Aehre bildend, die Kelcbe sind wea niger rauhbaarig, sondern nur bie und da mit kurzen krausen Härchen besetzt; dagegen zeigen sich zahlreiche harzige Punhte; die Einschnitte sind weniger tief, die Corollen bleiner and die Staubfiden immer über diese hinausstehend. Der Geruch ist sebr stark and insbesondere bei weitem stärker, als bei Meatha silrestris, unter welchem Namen sio wahro scheinlich $\mathbf{S m i t h}$ in dem Prodromus florae graecae anfïhrt.

Diesen Bemerkungen gemäls ist höchst wahrscheinlich die Mentha niliaca des wahre Sisymbrium des Dioscorides, von deren starkem Geruche schon der alte Pharmalsologe redete, and somit eine uralte Arzneipflanze, die die Beach. tung der Aerzte verdient.

Mentha tomentella Flor. portug. fand Hr. Prof. L. sehr häufig in Zante, und nicht selten im Peloponnes; sie ist der Mentha Pulegium nahe verwandt, von der sie jedoch im $\mathbf{H a}$ bitus abweicht. Sie wächst auf Bergen und hat weit umber kriechende Stengel, die wie die Blätter in jenen Gegenden niemals glatt sind.

Dioscorides gab keine Beschreibung ron ceinem Pan legium, sondern sagt nur, dafs es eine überall bekannte Pflanze sey.

Salvia pomifera, wird nach dem Berichte des Hrn. Apo. thekers Landerer von den heutigen Griechen bei Erkältungskrantheiten gebrancht. Auch diese Pflanze war schon 
im Altertbum bekannt, and wird bereits ron Theophrastus ron Eresos angeführt.

Leontodon gymnanthus Link: radice lanigera, foliis runcinatis, floribus praecocibus (ante folia), peranthodii phyllis interioribus binerriis. Hänfig im Peloponnes um Nauplia, Poron u. s. w. Eine sehr interessante, dem Leontodon Taraxacum ganz ähnliche Art, die sich vorzüglich dadurch unterscheidet, dafs die Blumen ror den Blättern erscheinen.

Pyrus cureifolia Gussone, ein Strauch oder bleiner Bum, den Hr. Prof. L. bäufig an den felsigen Bergen des Peloponnes, zumal zwischen Nauplia und Corinth sab; er glaubt, es sey Pyras communis var. $\beta$ Smith Flor. graec. and P. communis Exp. Mor., dürfte jedoch eine gute Art seyn, die schon sehr deatlich durch ihre riel schmäleren Blätter sich unterscheidet. In Sicilien bei Syracus, so wie in Sardinien sah Hr. Prof. L. denselben Strauch, dessen Früchte, wonn sie teig (puteidus) geworden sind, gegessen werden.

Lotus argalicus Link: Caule fruticuloso glabrescente, foliis absescilibus, foliolis oblongis apiculatis, pedunculis longis subtrifloris, carina acuta, leguminibus teretibus subdepreasis apiculatis.

Diese neue Art, ein kleiner gestreolter, nm Nauplia sebr bäufig wachsender Strauch, steht dem Lotas prostratus Desfont. am nächsten, und ist, wie Hr. Prof. L. nachweist, der so berühmte und vielbesprochene Lotos des Homer.

Spartium junceum, ist nach Hro. Apotheker Landerer ein Hausmittel der Griechen, wovon sie ein Decoct tassenweise gegen Dysarie anwenden. Es ist diefs abermals eine uralte Arzneipflanze, ron welcher Dioscorides ausführlich redet, und die zumal durch ihre Purgirkräfte sich auszeichnet. Astragalus creticus Lamark Wächst nicht selten auf derı hohen Gebirgen des Peloponnes, namentlich um Corinth, auf dem Berge Oleno und anderwärts. Nach dem Berichto 
des gewadten Pharmacenten Hra. Gaanacopulo in Patras wird das Traganth-Gummi auf dem Berge Boidia und sonst bei Patras gesammelt, sodann nach Patras und von da nach Trient transportirt. Ueber die Bentimmung diecer Pflanze sind die Botaniker nicht ganz einig, wefshalb Hr. Prof. L. die Angaben der Flore graeca ron $\mathbf{S}$ mith mittheilt, dort heifst es:

Astragalus aristatus Willd. synon. A. sempervirens La-

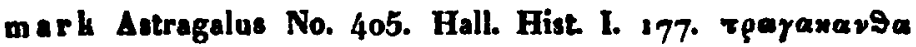
hodie in Peloponneso, und dann wird am Ende zugesetzt: Wächat sobr büufig anf den Gebirgen des Peloponnes, such anf den Gebirgen Thessaliens und der Insel Cypern. Von diesem Strauche wird das Traganthgummi jährlich ans Patras nach Itạlien gebracht. Dann folgt

Ao creticus Lam. mit Tournefort's Synonymen. Sib. thorp fand sie auf Sandhügeln, auf dem Olymp in Bithynien auf den sphakiotischen Bergen in Crete. Diese Species liefert auch Traganth.

In der Exp. Mor. 213. wird Astragales aristatus nur auf Sibthorp's Autorität hin angegebon, and eben so beruht daranf die Benennung in der getrockneten und bänflichea Sieberischen Sommlung.

Hr. Prof. L. glaubt aber, dals $S$ mith die Species ver. wechselt und sich gänzlich geirrt habe. Der Astragalus, welchen derselbe im Peloponnes fand, oder auch ron dem dortigen Botaniluer Hrn. Berger erhielt, hommt mit der Decan do ll ischen Beschreibung des A. creticus sehr gut überein. Der A. aristatus aber, den er auf den südlichen Alpen, zumal auf dem Mont Cenis sammelte, weicht von dem griechischen webr ab, eben $s 0$ ist A. aristatu Herbarii siober eine ganz andere Pflense. Dem A. cretiens steht A. siculus Bivon., den Hr. Prof. L. auf dem Aetna häufig «ah, und der Dorno wogen nur mit Mühe sammeln loonte, eehr nahe, weicht aber is der Structur des Kelches bedentend ab. Ob A. creticus 
Sieber mit der gleichnamigen des Lamark einerlei sey, wagt Hr. Prof. L. nicht su beutimmen, da io seinem Exemplare die Blumen mangeln. So viel ist aber gewifs, und für die medieinisch-pharmaceutiscbe Botanik wohl za beachten, dafs die ans Griechenland eingeführte Tragantheorte (MoreaTraganth) von Astragalus creticus Lamark eingesammelt wird.

Ueber die Bereicherangen der medicinischen Botanik durch die Franzosen, welche in einem bostbaren Prachtwerke (Expedition de Moree) enthalten sind, werde ich näch. stens Bericht erstatten.

III. Nachrichten von Arzneipflanzen und vegetabilischen Droguen verschiedener Länder.

A. Gewächse aus der Abtheilung der Exogenen oder Dicotyledonen.

Neapolitanische Angelica-Wurzeln.

Die wahre Angelica der Officinen ist eine Pflanze des Nordens, die in Schweden und Lappland an Flufsufern, an Gräben und auf feuchten Wiesen wächst; im südlichen Dentochland findet sie sich nur auf hohen Alpen und Gebirgen; es war darum irrig, wenn man bisher glaubte, dafs diese aromatische Dolde auch im südlichen Italien wachse. Selbst der berühmte Te nore führte im Jahre 183, noch Angelica Archangelica in seiner Sylloge plantarum uater den Pflanzen des Königreichs Neapel auf, and erst im Nachtrage zu diesem Wertie berichtigte er den Irrtbum. Die Dolden, die dort unter dem Namen Angelica in die Officinen kommen, sind aber nach neueren genaueren Bestimmungen die nachstehenden:

a. Angelica verficillaris $L$. oder Imperatoria verticillaris Decandolle; sie ist eb, die zuerst Tenore als Angelica 
Archangelica in seinen Bchriften sulfübrte; aie wächst in den Gebirgethälern der nördlichen Gegenden des Konigreichs Neapol; es gibt davon rerschiedene Varietäten, wovon nur eine, die Tenore Angelica Linnaei nennt, im Neapolitanischen rorbommt. Diefs wäre also die officinelle Angelica der neapolitanischen Botaniker. Man cultivirt aber in den dortigen Gärten noch eine andere Dolde, die Hr. Prof. T e no re defshalb Arohengelica culle Hortulanorum nennt; sie ist ganz glatt, çchön grün, und hat eine Aeischige süfse Wurzel. T. bringt sio als Varictät zu Angelica silvestris, bringt aber als Syno. nym Angelica sativa Miller hinzu, die ron den deutschen Botanikern za der wahren Archangelica officinalis gezäblt wird. Ohne Ansicht der Pflanze selbst läfst sich nun nicht wobl sagen, was die neapolitanischen Apothelier als Angelica in ihren Gärten ziehen; allein so viel ist unbezweifelt, dafs die wahre nordische Arzneidolde in jenerm warmen Klima hnum ein Mittel liefert, das dem unsrigen gleich zu achten ist.

b. Angelice nemorosa Tenore: caule superne pedancu. lisque pabescentibus, foliis tripinnato-sectis, segmentis ovatis abbreviatis obtusiusculis serratis, basi decurrentibus, in rolecro nallo, mericarpiis ellipticis, alis latitadine costes dorsales apice cristatas aequantibus.

Diese Dolde wächst in feuchten Thälern und Wäldern und sonst an nassen Orten, im Valle di S. Rocco, Agnano. Thre Wurzel wird Bracula genannt, and gilt als ein ganz vorzügliches Mittel bei chronischen Hautkranbheiten; sio ist charf und hat, so wie die Saamen, einen bockartigen Gerach, der bei der verwandten Angelica silvestris angenehmer gewürzhat ist.

In Sicilien wird die Wurzel von Heracleam cordatum als Angelica gebraucht, wie ich schon im Jahre 183، (Magaz. für Pharm. Bd. 33. S. 943.) anzeigte; später hat Hr. Virey in Paris (Journ. de Pharm. Jain 1835) dieselbe Sache erörtert. 


\section{Neapolitanische Gichtrosenwurzel.}

Schon vor mehreren Jahren (Magaz. für Pharm. Bd, 11. S. 105 u. d. f.) sucbte ich nachzuweisen, dafs unter dem Namen Radix Paeoniae zu verschiedenen Zeiten und an verschiede. nen Orten die Wurzeln ron mehreren wohl za unterschoidenden Arten in die Officinen gebracht wurden; diejenige, welche Herr Prof. Tenore anfangs in seinen botanischen Scbriften Paeonie officinalis nannte, bestimmte er später als Pueonia paradoxa; sie ist sebr nahe verwandt, wenn nicht identisch, mit derjenigen Pflanze, welche ich damals als Paeonia officinalis Herburii Linnuei beschrieb, und anch eine Abbildung beifügte, worauf ich bier verweisen 20 können glanbe.

\section{Herba Chamaepitys.}

Der verstorbene Geiger sagt mit Recht ron diesem Krante: Herba efficax hodie inmerito fere obsoleta; die alten deutschen Botaniker bannten es unter den Namen Schlaghraut, Foldcypresse, Erdweihrauch u. s. w.; als Mutterpflanze wird gewöhnlich Teucrinm Chamaepitys $L$. angegeben, die aber zwei Arten begreift, welche erst Schreber genauer unterschied, nämlich:

a. Ajuga chia Schreber: floribus axillaribus folia suporantibus, caule diffaso.

Diefs ist die im südlichen Europa gemeinere Form und höchst wahrscheinlich die wahre Chamaepitys der alten griechischen Aerzte; selbst Tenore führte sie noch früber als Teucrium Chamaepitys auf, wie mehrere andere italienische Floristen, so dafs es nicht unwahrscheinlich ist, dafs gerade diese Art noch jelzt in den Officinen des südlichen wärmeren Europa sich vorfindet.

b. Ajuga Chamaepitys Schreber: floribus axillaribus colitariis folio brevioribus, canle ramoso erecto. 
Nur diese Art ist diesseits der Alpen zu finden, und sie ist daher durchgängig die Chamaepitys der deutschen Apothehen.

\section{Krausemünze-Arten.}

Bereits im Spätjahre 1829 suchte ich in einer Abbandlung, die bei der botanischen Section der in Heidelberg versammelten Naturforscher und Aerzte vorgelesen warde, za zeigen, dafs unter dem Namen Mentha crispa in den Apa. theken ganz verschiedene Pflanzen rorkommen, von denen viele unter besonderen Namen als eigene Species beschrieben wurden, aber nach den damals auseinandergesetzten Grundsätzen nur als Varietäten in den Pflanzensystemen ihre Stelle finden können; eine Ansicht, die seitdem auch von mehreren andern Botanikern als die richtige befolgt worden ist. *) Auch Tenore führt ewrei Krausemünz-Arten, die im Königreich Neapel wild wachsea, an, die aber aus gleichen Gründen nur als Abarten untergebracht werden Hönnen. Es sind:

Mentha Lamarckii Tenore: spicis oblongis, rerticillis approximatis, foliis cordatis serratis undulatis subsessilibus, dentibus divergentibus cuspidatis, supra viridi-pabescentibus subtus incanis, calicibus pedunculisque birtis, staminibus corollam aequantibus, bracteis linearibas integerrimis.

Tenore fand sie an den Ofern des Vulturnus; er führt sehr viele Synonyme an, aus welchen man, trotz einiger Schwierigkeiten der Diagnose, sie nur für die Mentba cruciata des Lobelius and somit für eine krause Form der Menthe

-) Bemerkungen über die Gewächse, welche man mit dem Namen Mentha crispa bezeichnete. Magaz. für Pharm. Bd. 29 S. 154 u. d. f. Man sehe ferner meine Uebersicht der officinellen $\Delta r$ ten der Gattung Mentha in Brandes Archiv Bd. 32. S. 195. 
rotundifolia halten kann; auch ist gerade diese Form die Mentha crispa der Franzosen, and überhaupt in den südlichen wärmeren Gegenden eine der verbreitetsten Formen. Irrig zieht Te nore die Mentha crispata Schrader dahin, welche ungezweifelt eine krause Form der Mentha viridis Lin. naei ist.

Montha serotina Tenore: foliis ellipticis breviter petiolatis sapra scabris subtus canescentibus dentato-undulatis, spicis abbreviatis o verticillis approximatis compactis, staminibus inclusis.

Eine Varietät dieser Pflanze: foliis utrinque canescentibus undulatis serratis incisis, staminibus exsertis, beschrieb Tenore früher in der Flora neapolitana unter dem Namen Mentba crispa; er fand sie an überschwemmten Orten und Gräben in verschiedenen Gegenden des Königreichs, die er genau anführt.

Diese krause Münze der Neapulitaner scheint ganz rollständig dieselbe zu seyn, die man in Heidelberg, Mannheim und der Umgegend ebenfalis in den Aputheken vorfindet; es ist die Mentha Balsamita Lobelii und somit eine krause Form der breitblättrigen $\mathbf{A b a r t}$ der Mentha silvestris (nemorosa), was man um so eher annehmen darf, ale Tenore selbst in der Beschreibung seiner Heise nach den Abrazzen genau diese Krausemünze als Mentha silvestris nemorosa beschrieb. - Wenn man bei uns diese krausblättrigen Labiaten our in Gärten, in südlichen Italien aber im Freien findet, so mag wohl das wärmere Klima seinen Aatheil an der Crispation Jer Blëtter baben.

Noch bemerkt 'Tenore, die wahre Mentha crispa Linnaei habe er Anno 1824 in dem Wiener botanischen Garten, der unver Host's Aufsicht steht, cultivirt geseben; auch habe er sic nachler in Neapel selbst gezogen und immer rou 
der Mentha Lamarctii rerschieden gefunden, wefshalb er folgende Diagnose beifügt:

Mentha crispa: spicis subcapitatis, calicibus pedunculisque glabris, foliis cordato.orbiculatis glabris undulato-crispis dentatis, dentibus cuspidatis, bracteis inferioribus laciniatis.

Darin wird man wohl nichts anderes erkennen Können, als die uraite Mentha crispa des Valerius Cordus, oder die Mentha bortensis $\mathbf{O p i t z}$, die ibrerseits nichts anderes seyn dürfte als eine krause Form der Mentha aquatica.

Ueber die Gattung Mentha äberhanpt, and iber die Men. thae crispae insbesondere ist rorzugsweise die klassiscbe Monographie von Bentham nachzulesen, woron in dem schwodischen Jahresberichte für Botanik vom Jahre 1834 ein Auszog enthalten ist; es kann mir nur angenehm seyri, dafs dor scharfsinnige Britte, der meine Abbandlang rom Jahre 1829 kaum gelesen hat, fast in allen Punhten mit meinen damals geäufserten Ansichten und Anordnungen übereinstimmt.

Schon früher (Annalen Bd. 14. S. 113.) warde erinnert, dafs in Ostindien Mentha Pudina Hamil ton als Krausemünse cultivirt wird. Nach Henschel ist die ron Rumphins beschriebene Mentha crispa ( 5 alassi) Ocimuna menthoides L. ").

Ursprung und Mutterpflanze der Cassia fistula.

Es ist bekannt, dafe die Röhren-Cassie in Aegypten, so wie in Ost - and Westindion vielfältig cultivirt wird, und ron allen diesen Gegenden die Früchte zan Arzneigebrauche nach Europa verschickt werden; allein schon $\mathbf{R}$ u mphius und viele andere Botaniher erinnerten, dafs der Baum, welcher diese bekannte Drogne liefert, weder in Asien noch Amerika und

-) Man vergleiche auch Magaxin futr Pharmacie Bd. 33. S. x41. 
selbst nicht in Aegypten einheimisch ist, sondern aus dem inneren Afrika stamme. Wirklich entdeckte vor mehreren Jabren der gelehrte Reisende, Hr. Caillaud, in dem Theile Nubiens, der an den Nil grenzt, eine Art von wilder Cassia fistula, die dem cultivirten Banme vőllig ähnlich ist, nur mit dem Unterschiede, dals die Frucht eine grüne Pulpe hat, die eben nicht angenehm schmeckt. Hr. Delile, der die systematische Bestimmung der von Hrn. Caill a a d entdechten Gewächse übernahm, nennt diesen nubischen Banm Cassia Arerek; auch äufserte man die Ansicht, dafs dieselbe wirblich die Mutterpflanzo der officinellen Drogue seyn dürfte.

Man weifs übrigens, dals es in Amerika wie in Asien Arten von Cassia oder Cathartocarpus gibt, deren Früchte eine ähnliche Eigenschaft wie die gewöhnliche Cassia fistula besitzen. Bereits $\mathbf{R} \mathbf{m} \mathbf{m}$ hi as beschrieb deren mehrere, die noch nicht alle ihre Stelle in den Systemen der Botaniker gefunden haben. Am interessantesten ist Rumph's Cassia Fistula silvestris rabra, oder Cassia nodosa $R_{0}: b_{u} \mathrm{r}_{\mathrm{g}} h$, deren Früchte denen der officinellen Cassia fistula ganz ähnlich sind, nur sind sie etwas länger, nngefähr zolldich, und bei der Beife aufserbalb schwarzgrau, gegen den Stiel hin geLrümmt und schmäler; ihre Schale ist rauher und dicker als bei der gewöhnlichen Art, auch da und dort gleichsam eingeschnürt. Die Pulpe der Cassia nodosa ist trocken, weifslich, zähe und schwammig; die Saamen flach, zusammengedrückt, glänzend, safrangelb oder schon braan und in der Mitte von einer Furche durchzogen.

\section{Zu Brenn-Cylindern oder Moxen taugliche Pflanzen.}

Man weifs, dafs die jetzt meistens aus Baumwolle gefertigten Brenn-Cylinder schon seit alten Zeiten im Orient gebräuchlich waren, uad da noch immer aus den Blättern einer Planze bereitet werden, ron der man lange glaubte, dafe oie 
nichts anderes als anser gemeiner Beifufs, Artemisia rulgaris $L_{\text {. }}$ ey. Nenere und genanere Untersuchungen lehrten aber, dafs die Pllanze, welche die chinesische Moxa liefert, eine davon verschiedene und eigene Art ist, welche der behannte rusciache Botaniker Besser mit dem Namen Artemisia Moxu bezoichnete. Eine sebr ausfübrliche Beschreibung derselbea lieferte schon der alte Romphius, aus dessen klassischem Werke die nachstebenden Notizen entnommen sind. $\mathrm{Aumph}$ nennt sie Artemisia latifolia, und bemertt, dafs diels Gewächs, so lange es nicht blühe, ron der Matricaria sinensis, welches unser allbekanntes Chrysanthemum indicum ist, baum unterschiedea werden hönne; doch sejen dio Blätter der Artemisia länger, dünner, tiefer eingeschnitten, unten weifs und oben vielfach mit linsenartigen Flecken bezeichnet; auch bōnne man beide durch den Geruch unterscheiden. Der Stengel der An temisia Moxa ist ungefähr a Fufs boch und nackt und trägt zahlreiche bleine gelbliche Blümchen. Die Malajer nennen die Pflanze Baru 'Tschina d. b. chinesischen Zunder; sie wurde um das Jahr 1660 aus Java nach Amboina gebracht, wohin sie, wie man sagt, aus China gekommen seyn soll. Der Name Moxa ist japanischen Ursprungs und bedeutet nichts anderes als trockene Artemisia, die zu Brenn-Cylindern tauglich ist, während die frische Jomongi oder Nophonti genannt wird.

Die Bereitung der Brenn-Cylinder ist ganz einfach; man trocknet nämlich die Beifulk-Stengel im Schatten, und reibt sie dann zwischen den Händen zu feinen Fasern, aus denen die rauhen und groben herauszulesen sind. Diese feinen, der Banmwolle fast ähnlichen, Fäden tragen dann speciell dea den Namen Moxa

In Spanien dient eine andere Pflanze ans der Familie der Synanthereen, nämlich Echinops strigosus $L$., zu gleichem Zwecke, indem man sowobl Zunder als Brenn-Cylinder oder Moxen daraus bereitet. 


\section{Ballota lanata.}

Von dieser auch in Deutschland schon riemlich bekannten Pllanze lieferte Jori eine weitläufig beschriebene chemische Analyse, woza er, wie es scheint, besonders durch die grolsen Lobsprüohe veranlafst wurde, welche Brera dieser Planzo ertbeilte. Sie enthält nach dieser Untersochung: Gerbestof, welcher die Eisenoxydsalze grün färbt, eine bittere aromatische harzige Substanz (Picroballota), grünes Pflanzenwachs (Chlorophyll), Chlornatrium, salpetersaures Rali, Eisen, wahrscheinlich als Protoxyd., Thonerde, Halkerde. Sämmtliche Bestuadtheile, das Chlorophyll ausgenommen, bilden mit dem überschüssigen Gerbestoff in Wasser lösliche Verbindungen. Die von Brera rorgeschlagene Bereitungsmethode, mittelst sobwachen Weingeistes die wirhsamen Bestandtheile aus der Pflanze zn ziehen, ist folglich die zweckmölsigste. (Antologis med. 1834. Nov.; Sch midt·Jahrbücher 1836. Bd. 16. Heft 1. S. 5.

\section{Solanum Lycopersicum.}

Der Paradiesapfel oder Goldapfel hat bis jetzt in den europäischen Officinen kanm eine Stelle gefunden, obgleich or ron pinem nngrischen Arzte als ein ganz vorzügliches Mittel gegen das bösartige Blutgeschwür (Anthrax) empfohlen worde, *) Kürzlich theilte Doctor Bennet, Prof. am Med. Collegium des Erie-Sees, über diese Poma Amoris oder paradisiaca Folgendes mit. 1) Der Liebesapfel ist ein der hräftigsten eröffnenden Mittel in der Materia medica und bei allen Leberkrankheiten and Leiden anderer Organe, wo Calomel indicirt ist, dürfte kein Mittel wirksemer und gefahrloser befunden werden. 2) Ein chemisches Extract, das Hr. B. bereiten zn lassen gedenkt, dürfte den Gobrauch des Calomel

-) Neue Entdeckungen in der Materia medica 1828. S. 362. 
kưnftig ganz verbannen (? D.). 3) Heftigen Durchfall hat er mehrmals. blos mit Solenum Lycopersicum geheilt. 4) Als Nahrangsmittel genossen, verhindert ea fast ohne Auenahme Dyepepsio und Unverdaulichkeit. ( $\nabla$. Froriep's Notizen Bd. 46. No. 16. 1835; Sc hmidt Jahrbücher Bd. 10. Heft .. S. 20.)

Man mufs hierbei erinnern, daf die Früchte dieses Solanum in Deutschland nur in sehr warmen Sommern gehörig auswacbsen und vollhommen reifen, so dafs nur in wärmeren Ländern ein allgemoiner Gebrauch wird davon gemacht werden urnnen.

\section{Semina Spartii scoparii.}

Die Saamen des gemeinen Ginsters, Pfriemen - oder Becenurautes, liegen zu $\theta-12$ in ibren Hälsen; sie sind läaglich verkehrt eiförmig, am Grunde abgestatzt, gelblieh und ron widerlich bitterm Geschmacke. Hr. Dr. Richard Pearson in Birmingbam empfiehlt sie in einer eigenen Schrift als ein Mittel bei bydropischen Leiden ") and zieht mit ganz gutem Bechte den Gebrauch der Saamen dem der übrigen Theile der Planze vor. Man soll dieselben oder auch die fast nierenförmigen Saamen des im südlichen Europa einheimischen Spartium junceum Lo (Spartianthus junceus Linh) in Form von Tinctur anwenden, und 2 war nach folgender Vorschrift: Rec. Seminum contusorum Spartii juncei Encias duas, Spiritus tenuioris Uncias oeto. Mecera per dies octo et cola. Auch hönnen die Saamen in Pulverform verordaet werden. Hr. Dr. P. hält die Ginstereaamen für ein toniuches Diureticua, welches fortwährend gebraucht werden töane, und die Squills, so wie die Digitalis ersetze. Ein deutscher

-) Observations on the action of the Brwom seed in dropsical af: fections. London 8835 . 8. Mit 3 Kupfertafeln. 
Recensent, Hr. Dr. Vetter, dieser Schrift stimmt (Schmidt's Jahrbücher Bd. 10. Heft 3. S. 36ı.) dieser Ansicht nicht bei, und meint dagegen, wenn die Nützlichleit der Ginstersaumen sich bestätigen sollten, so könnten sie hø̋chstens den Baccis Juniperi an die Seite gestellt werden,

Ref. lann weder die eine noch die andere Annahme als eine richtige ansehen, da sämmtliche genannte Planzen nur in dem einen Pankte miteinander übereinstimmen, dafs sio nämiich die Diurese befördern; die Saamen ron Spartinm haben, was schon die alten Aerzte wufsten, zugleich eine emetische und purgirende Kraft, die ron dem Catbartin ab. hängen dürfte, und dadurch unterscheiden sie sich wesentlich von den Wachholderbeeren. Spartium ist eine Pllanze der Leguminosen, Juniperus der Coniferen, erstere bat einen bittern Extractivstoff, letztere ätherisches Oel als vorherrschenden Bestandtheil; Spartium ist ein Diureticum purgens, Juniperus ein Diureticum excitans. ")

\section{Mikania Guaco.}

Da noch immer die Brechruhr in Deutschland nicht ganz verschwunden ist, so dürfte es ganz an der Zeit seyn, die neuesten Erfahrungen zu erwäbnen, nach welchen diese mexibanische Pflanze aus der Familie der Compositen ein ganz vorzügliches Mittel gegen die Cholera asiatica ist. Ed. de Cbaniac, Officier der französ. Marine, berichtet darüber Folgendes: Als die Brigg Adonis bei ihrer Fabrt von Haranna nach Mexilto im Jahr 1833 in Vera-Cruz anlegte, bokam sie die Cholera, welche damals in Mexiko herrschre, an Bord. Unter allen dagegen angewendeten Mitteln bewies sich das Guaco am wirksamsten, ja seine Wirkungen waren so

-) Man vergleiche Uebersicht der Diuretica in den Heidelberger medicinischen Annalen Bd. 2. Heft ı. S. 1-35. 
Tunderbar, dafa man es für ein wabres Specificum gegen dio Cholera halten Hōnnte. Das Mittel wirkt insbesondere auf das Herz and den Kreislauf, den es bethätigt. Alle Cholera-Kranke, die es gleich im Begion des Uebels gebrauchten, wurden gerettet, und selbot ron denen, hei welchen die Cholera schon einen gewissen Grad erreicht batte, wurden die meisten erhalten, sobald eine freie und röllige Reaction eintrat. (Ga. zette med. de Paris No. 28. 1835.)

Dr. Chabert, Arzt am Militärspitale in Mexiko, gibt in einfachen Fällen alle halbe Stande eine bleine Tasse einer warmen Guaco-Abkochung, bis allgemeiner Schweifs und eine gebơrige Hautwärme eintritt, die man einige Tage unterbält, indem man allmählig das Guaco wegläfst. Zum Löschen des Dorstes gibt man die Ablochung mit 2 Drittel oder der Hälfte Wasser. In den gefährlichsten Fällen, bei Cholera algida, Kaltwerden, Verlust des Pulses a. a w., vermischt man mit einem Löffel der weingeistigen Tinctur 6-8 Löffel Wasser and läfst alle $1 / 4$ Stunde abwechselnd a Löfel voll ron dieser Mixtur und eine hleine Tasse des Decocts nehmen. Hat sich der Puls gehoben, ist die Wärme wiedergehehrt, hat sich die Transpiration wieder eingestellt, so läfst man die Tinctur weg und gibt blos das Decoct fort, jedoch in grüfseren $\mathbf{Z}$ wischenräumen. In der Mehrzabl der Fälle tritt nach dem Aufbören der Cholerasymptome Scbmerz im Epigastrium, brenneuder Durst ein; in diesem Falle beseitigt die mit der Hältte oder a Drittheilen Wasser rerdünnte $\mathbf{A b k o c h u n g}$ die Zeichen von Reizung, den Schmerz und den Durst. Wird die $\mathbf{A b}$ hochung vom Magen nicht vertragea, so gibt man sie im Klystier. Die allgemeinen und rrtlichen Blutentziehungen, die äofseren Mittel finden wie sonst ihre Anwendung, allein innerlich darf neben dem Gaaco kein anderes Mittel gegeben werden. Zur Ablochung nimmt man 2 Drachmen Stengel und $1 / 2$ Drachme Blätter auf $1 / 2$ Flasche $W$ asser, bis anf eine 
Flasche eingelsocht. Die Ablsochung ist bitter, os mafs übrigens diese Tisane mehrere Stunden kochen, und es hann das nämliche Holz zweimal gebraucht werden. Die Gaaco-Tinctur wird, wie alle andern Tincturen, mit Weingeist durch Aufguf bereitet; ibro Farbe ist grün. (Schmidt Jahrb. der gesammten Medicin Bd. 11. Heft 1. S. 17.)

\section{Matica oder Matico.}

Hr. Aimé $\mathrm{Aul}$ zu Antwerpen theilte über diese neue Arznei-Drogue folgende Nachricbten mit: Die gevannte Planze gehört nach Mérat und de Lens zu den pfefferöbnliohen Gewächsen, mit langen, wechselseitig stehenden, geaderten, unten behaarten Blättern. Zwischen den Händen gerieben rerbreiten dieselben einen leicht aromatischen, der Menthe ähalicben Geruch; ibr Geschmack ist anfangs indifferent, apäter scharf bitter, aber nicht zusammenziebend. Man könnto sie, ihrem äufsern Ansehen nach, vielleicht mit der Salvia oficinalis verwechseln. In Peru gilt die Planze für ein n̈uíserst liräftiges Adstringens, seibsi das trockene Pulver derselben soll im Stande seyn, Blutungen aus den gröfsten Gefälsen za stillen (?). Das Pulver braucht man auch ioner. lich, da man den Absud für luraftlos hält. Dr. So mm é zu Antwerpen, Oberarzt der chirurg. Abtheilung des dasigen Hospitals, machte einige Versuche mit diesem Mittel in blutigen und schleinigen Prolluvien, aber obne besondera Erfolg. Dagegen zeigte es sich dem Dr. Van Haesendonch dasel hat bei chronischen Lungentatarchen und Lungen-Phthisen mit übermälsiger Expectoration obne fieberhafte Aufregung, als ein sehr schätzbares Mittel. In Fällen dieser Art verringerte sich die Menge des Auswarfs in 24 Stunden oft am die Hälfte, uad bald hōrte derselbe ganz auf, so daf die Patienten nach karzer Zeit das Hospital wieder rerlassen bonnten. In vielen Fällen beschwichtigte das Mittel selbst das bereits 
eingetretene beltische Fieber, die Nachtschweifse a. * $w$. Zum innerlichen Gebrauche gibt man pro dosi 1 Scrupel bis 1/2 Drachme des Pulrers der getrockneten Blätter, alle ₹ Stuaden wiederholt. Oder ein Electuarium: Rec. Pulveris herbae Maticae Unc, unam vel duas, Syrupi diacodion q. a nt f. Electuar. D. S. Theeloffelwoise 20 nebmen. Es werden 4 Krankbeitsfälle mitgetheilt, welche die Matica bezwang: a Fälle beginnender Rhthisis, I Catarrhus chronicus und I Pneumonia chronica.

In Hambarg soll näohsten eine Sendang dieser Planze ankommen, so dafs man auch von deutschen Aerzten Versache mit diesem neuen Arzneimittel erwarten dart. (Annales de Medecine belge Février 1836; Schmidt Jahrb. Bd. 11. Heft 2. S. 1.54.)

\section{Lindenblüthe.}

Von verschiedenen Seiten her hat man daranf aufmertsam gemacht, dafs es nicht gleichgültig seg, ron welcher der gemeinen deatschen Linden-Arten die Blumen zum officinellen Gebraacbe eingesammelt würden; eine Sache, auf die man früber um so weniger achtete, da Linné alle in Earopa wildwachsende Tiliae nur ais eine einzige Species anerbannte, der er darum den Namen Tilia enropaea gab. Neuere Botanilher haben diese T. europaea in mehrere Arten zerspalten, und besonders fübrt Host in der zweiten Ausgabe seiner Fiora Auntiaca eine ganze Reihe nener Linden auf, zwar mit Disłnosen und Bescbreibungen, aber ohne alle Synonymio, so dafs es am so schwieriger ist über sie zn urtheilen, da thein anderer Standort, als die Alleen und Anlagen in and an Wien nambeft gemacht wird.

Reichenbach in seiner Flora germanioa excursoria führt 14 Arten an, worunter ihm jedoch selbst nehrere zweifelhaft sind, behält abor als besondere Species eine Tilia eu. 
ropaea L. bei und bezeichnet diese als die officinelle $\Delta r t$, von welcher die geruchvollen Blumen gesammelt werden sollen; ais Synonyme fügt er ihr bei 'Tilia vulgaris $\mathbf{H}$ a y a e, T. Tecksiana Joh. Ba ahin und ' $\mathrm{T}$. intermedia Decandolle. - Die Blumen von Tilia platiphyllos Scopoli oder T. panciflora Hayne sind nach ihm ebenfalls wohlriechend, dagegen die von Tilia parvifolia Ehrh. u. Hayne als geruchlos angegeben werden. - Mehrere Sommer hindurch habe jch mich mit dieser Sache beschäftigt, ohne zu einem reinen Resultate gelangen za können. Da es aber zurörderst darauf ankommt, sich über die anzunchmenden Arten von europäischen Linden za verständigen, so dürfte es den Lesern angenehm seyn, eine karze Uebersicht der neuesten Bearbeitung dieses Gegenstandes hier zu erhalten; es ist diefs die Revisio Goneris Tiliarum, auctore Eduardo Spach, nach welcher die Linden in zwei Haupt-Sectionen zerfallen, nach folgenden Merkmalen:

\section{Sectio prima.}

Staminodia nulla. Stamina $25-45$, petalis longiora, stylum etiam post anthesin superantia; filamenta vix apice bifurca, basi nunc libera, nunc pentadelpha, phalangibus 5 8 andris, petalis antepositis, cum filamentis $1-3$ liberis alternantibus. Nux aut chartacea, fragilis, costis filiformibus, aut lignosa, costis prominulis.

Alle Arten dieser Abtheilung sind in Europa einheimisch.

A. Mit glatten Blättern, Blattstielen und Zweigen. (Nur auf der untern Seite der Blätter in den Venenwinkeln siod Bartbaare.)

a. Mit einer schiefen, papierartigen, brüchigen Nafs, deren Rippen fadenförmig und bisweilen von einem filzigen Gewebe ganz verhüllt sind.

Tilia silvestris Desfontaines. Es gibt davon mehrere Varietäten, insbesondere eine mit ovalen Blättern, ovalifolia 
seu minor, durch frübe Blütbe ausgezeichnet; dann eine mit herzförmigen Blättern, cordifolia seu major, dahin gehört Tilia parvifolia Guimpel et Hayne; endlich eine mit runden Blättern, rotundifolia, wozu Tilia microphylla Ventenat als Synonym gehört.

b. Mit einer lederartigen, etwas schiefseitigen Nufs, deren Rippen hervorstehen.

Tilia intermedia Decandolle, wozu $\mathrm{T}$. europaea $\mathrm{Smith}$, T. vulgaris Hayne, T. Tecksiana Bauhin, und als Bastard T. hybrida Becustein.

Reichenbach sagt von der Frucht dieser Art: Capsula sublaevis regularis, obsolete pentagona. Spach dagegen: Nux subturbinata vel oblique obovata, umbonata, velutina; noch setzt er hinza: nux $3-4$ lineas alta, diametro $21 / 2-3$ lineas sublignosa pentagona, tomento floccoso lutescente induta, demum glabrescens.

Dagegen sagt er bei der T. silrestris: Nux nisi minoris volumine, tenuis, fragilis, tomento rufescente floccoso, demum deciduo induta, immatura turbinata vel pyriformis.

B. Mit holziger Nufs, deren Rippen sebr hervorstehen. Die jüngeren Zweige, Blattstiele, and die untere Seite der Blätter sind sämmtlieh mehr oder weniger mit rauhen Härchen besetzt.

Tilia mollis $S_{p} a c h$. Dazu gehören Tilia cordata Miller, T. cordifolia Besser, T. europaea Desfont., T. corinthiaca Bosc. a. s. w.; sie ist im mittleren und südlichen Europa einheimisch, die Blätter sind auf beiden Seiten fein behaart, die Gestait der weichbehaarten Frucht variirt sebr. Spach gibt folgende Varietäten an:

valgaris: dahin T. platyphyllos Ventenat und T. pauciflora Hayne.

plurillora. Jeder Blumenstiel trägt $5-7$, bei der vulgaris nur 2-3 Blümchen. 
longepetiolata, die langgestielte.

bracteosa. Mit ausgezeichnet langen Nebenblättern.

leptolepis. Die Nebeablätter sind ungewöbnlich kurz, schmal, lanzettförmig.

brevipes. An den ganz kurzen Blumenstieichen benntlich. corallina. Die jungen $\mathrm{Z}$ weige sind entweder das ganze Jahr hindurch, oder nur im Winter bluthroth. Dabin gehören Tilia europaea Hooker, T, rubra Decandolle. Sectio secunda.

Flores staminodiis praediti. Stamina 40-80 petalis bre. viora: tilameata semper apice conspicue bifurca, basi irregulariter polyajelpha marginibusque staminodiorum adnata. Stylus post unthesin stamina superans. Nux lignosa costis filiformibus vel ovanidis. Eynue $7-3$ florae. Folia saepissime illis specierum sectionis primac najora.

Diese Abtheilur: bestelt aus fünt amerikanischon und cinar curopäisches A:t. Diese letztere ist Tilia argentea Desfont., welche auch unter den Namen Tilia alba Waldstein, $T$, rolundifelia Ventenat und $T$. tomentosa Mönch vorkommt. Sie wächst wild in Ungatn, Sieberbürgen, Slaronien und Croatien. Man darf' sie nicht mit der amerikanischen Tilia alba Michanx oder ' $T$. heterophrila Ventenat rerwechseln.

Fon der Siberlinde stammen rielleicht die so avfserordentlich lieblich und angenehin riechendeu constantinopalitanischen Lindenblüthen, wovon Apotheker I, anderer in Athen cine Schachtel voll zur Probe nach Deutschland schichte. Der jüngere Bachrier in München stellte mit ihnen einige Versuche an, um das liebliche Arom derselben zu isoliren. *)

Noch folgt hier dic Clavis analytica Tiliarum nach $\mathrm{Spach}$, durch deren Gülfi alle Arten sehr leicht bestimmt werden

7) Brandes neues Arihiv Bd. 8. S. $7^{5}$. 
Können. Der Gebrauch einer solchen Clavis ist wobl den Lesern schon zareichend behannt, so dafs es unnöthig ist, darüber ein Wort zu verlieren.

Staminodia nulia. Stamina petalis longiora, stylum etiam post anthesin paulo superantia. Nux vel fragilis, costis

1. filiformibus, rel lignosa, costis prominalis . . 2. Flores staminodiis 5 praediti. Stamina petalis breviora. Stylus post anthesin plus minusve exsertus. Nux costis tenuibus vel eranidis, semper lignosa . . . . 4 .

2. Folia subtus plas minuspe hirsuta . , T. mollis. . Folia praeter barbulas paginae inferioris glabra . 3. Nux coriacea, subaequilatera, costis prominuiis. Folia 3. supra laete viridia, subtus pallida vix glauca . T. inttermedia. Nux chartacea fragilis obliqua, costis filiformibus. Folia supra obscure viridia, subtus glauca . $T$. silvestris. Gemmae glabrae lucidae majores. Foliorum lamina praeter barbulas paginae inferioris glabra, v. pilis sinplicibus 4. pubescens. Filamenta staminodiis paulo breviora . 5 . Gemmae tomentosae vel pubescentes parrae. Foliorum pagina interior pilis stellatis tomentosa $\nabla$. pubescens. Filamenta staminodiis subduplo breviora, . . 6 .

Folia marginibus puberula, subtus praeter barbulas gla-

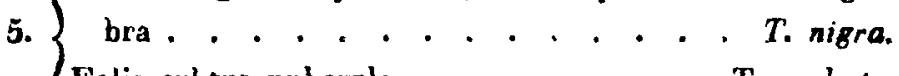
Folia subtus puberula . . . . . . . T. neglecta.

Foliorum superioram petiolus laminae subaequilongus. Stylus glaber. (Barbulae foliorum ferrugineae.)

6.

Foliorum omnium lamina petiolo triplo-quintaplo lon(gior 
(Folia subtus albido tomentosa, in axillis renarum im7. berbia. Nucis costae prominalae . . T. alba. Folia subtus incana $\checkmark$. puberala, in axillis renarum barbulata. Nucis costae evanidae . . . . - 8. Folia sabtus incano-velotina. Cymae dense multiflorae. Petala apice obsolete crenulata, stylo paulo superata.

8.

Folia subtus tenuissime puberula. Cymae laxe multiflorae, subpaniculatae. Petala apice profunde emarginata, stylo demum longe superata . . . . . T. laxiflora. (Annales des Sciences naturelles Vol, 2. p. 331 ff.)

\section{Columbo - Wurzel.}

So lange diese Wurzel in den Officinen belsannt ist, 80 lange danert auch die Klage, dafs sie mit andern falschen Wurzeln untermengt im Handel rorkomme. Mag diefs nun absichtlich gescheben oder auch nur zufällig sich ereignen, 80 bleibt es immer eine unangenehme Sache, dafs bei einer ron den Aerzten so geschätzten und so viel gebrauchten Drogue es fast nöthig wird, jedes Stüclschen genau zu betrachten, um von seiner Aechtheit versichert zu seyn. Besonders hat der verstorbene Stromeyer bei Gelegenheit der Apotheken-Visitationen im Hannörerschen mehrere falsche Columbo-Wurzeln wahrgenommen, die der Neffe desselbea in einer eigenen Dissertation beschrieb. Diese scheint nicht in den Buchhandel gekommen zu seyn, und aus ihr eatnimmt Ref. die nachstehenden Angaben.

Zuerst erwähnt S. einer falschen Columbo, die über Bremen in den Handel kam, and bereits 1820 ron Stoltze untersucht wurde. Diese Wurzel ist äufserlich bastanienbraun, innerhalb weifslich gelbroth, pulverisirt aber ron schön hell. gelber Farbe. Der Form nach kommt sie mit der wahren 
Colnmbo überein, doch sind die Stücle bäufiger in die Länge, als in Scheiben zerschnitten. Die Substanz besteht aus zwei Schichten, welche durch schwarze Linien nicht getrenut sind. Sie schmeckt anfangs süfslich, hernach bitter und widerlich, and riecht ungefähr wie ein Gemische ans Levisticum und Pimpinella. - Die ätherische Tinctur hat die Farbe des Franzweins, nicht das schön Goldgelbe der wahren; das Papier färbt sie etwas schmutzig braun und die Farbe beider wird darch Kali nicht geändert; sie schmeckt wenig bitter; mit Wasser gemischt entsteht nach einiger Zeit ein weilser und mit Bleizucherlösung ein kastanienbrauner Niederschlag. Lösungen ron salzsaarem Eisenoxyd und schwefelsaurem Eisenoxydul veranlassen in der Tinctur eine dunkelgrūne Fürbung; Galläpfeltinctar erzengt keinen Präcipitat. Das wässerige Decoct ist ganz durcbsichtig und hat die Farbe des Franzweios, schmeckt leicht bitter, und enthält nicht viel Schleim, obgleich I Drachme des Pulvers mit einer Unze Wasser die Dicke einer Latwerge annimut. Galläpfeltinctur veranlafet darin keinen Niederschlag und rectificirter Weingeist bewirkt eine reichliche Gallert-A bsonderung. Lösungen von Eisenoxyd und achwefelsaurem Eisenoxydul machen zwar die Farbe des Decocts dankler, veranlassen aber weder einen grünen noch einen schwarzen Niederschlag. Merkwürdig ist, dafs diese falsche Wurzel in Hitssicht der Reagentien zumal die Eisensalze sich zur wahren Columbowurzel eben so verhalten wio die falsche Angusturarinde verglichen mit der wahrea. Zwei Drachmen der falschen Columbo einem grofsen Hunde gegeben, verursachten Erbrechen.

Eine zweite falsche Columbo kam über England in den Handel und erscheint in Scheiben von $1 / 2$ bis a Zoll im Um. fang, und $1 / 4-1$ Zoll Diclse; auch sommen Stüclie 2-5 Zoll lang vor, wovon einige dem äufsern Ansehen nach Aehnlichkeit

Anasl. d. Pharm. XX. Bde. 3, Heft. 
mit der Radix Gentianae zeigen. Sie besteht nur aus zwei Schichten: denn aufserhalb ist die runzliche Rinde, die angefähr I Linie dick schmutaig braungelb, nach innen hellgelb ist; der innerste ron der Rinde umgebene Theil ist gelb, weich, biegsam, auf der Oberfläche nicht so runzlich, wie die wahre, sondern fast glatt enzufühlen, in der Mitte dünner und zusammengezogen, heller von Farbe and bei einigea Stücken durchlöchert. Diese Wurzel ist fast geruchlos und hat gekaut einen nur leicht bittern Geschmack. Dem Gewichte nach ist sie viol leichter als die wahre Columbo, ond verbält sich zu dieser ungefähr so, wie das Gewicht des Quassionbolzes za dem des Lignum Guajacum. Das Pulver hat eine gelbe Farbe.

Obgleich sorgfältig in einem Glase bewahrt, wurde die Wurzel doch sebr ron den Würmern zerfressen. Die ganze Wurzel in Indtinctur getaucht, zeigte seine Spur von Stärhmehl. Ein wässeriges, durch Digestion bereitetes Infusum war gelb, schmeckte bitterlich and seine Farbe wurde durch Kali causticum etwas erhöht. Das wässerige filtrirte Decoct war durchsichtig, gelb, bitterlich; Galläpfeltinctur, Weingeist, salzsaures Eisenosyd und lodtinctur brachten keine Veränderung in denuselben herror.

Eine bei mäfsiger Wärme mit Weingeist darch Digestion bereitete Tinctur war hellgelb, durchsichtlg, geruchlos, wenig bitter. Mit salzsaurem Eisenoxyd entsteht eine gelbgrüne, mit Kali cansticum eine dunkelgelbe Färbung; mit essigsaurem Blei wird sie ein wenig, ron Galläpfeltinctur aber nicht getrübt. Durch Abrauchen der Tinetur erhält man ein braangelbes, leicht bitteres, in Wasser ganz lösliches Extract; diese Lösung ist bellgelb, wird von Galläpfeltinctur nicht geändert, nimmt aber rom essigsauren Blei eine etwas hellere Färbang an. Nach Schrader gehört diese Wurzel entweder 
zur Gattnng Beta oder Rapa, mit denen sie noch am meisten Aehnlichlueit bat.

Eine fernere, ans Batavia eingebrachte falsche Columbo bestebt lediglich aus scheibenformigen, theils ovalen, theils randen Stücken, $2-5$ Zoll im Durchmesser und $1 / 4-1 / 2$ ZoH Dicke. Die Rinde ist sehr dünae, ranzlich, schmatzigbraut; der zunäohst von der Rinde umschlossene Wurzeltheil bildet nur eine Schichte, ist gelbbraun, und zeigt treisförmige Ausbreitungen; in der Mitte ist sie nicht dünn oder zusanmengezogen, wie die rorige Wurzel. Sie schmeckt sehr bitter and adstringirend and hat eiaen widerlicben Geruch; sonst ist sie nicht von Würmern zernagt, und das Pulver hat eine gelbgrüne Farbe.

Die ganze Wurzel in Iodtinctur getaucht, verändert ihre Farbe nicht. Das mit kaltem Wasser bereitete Infosum ist brann, sehr bitter, und wird die Farbe von ätzendem Kali nicht geändert. Das wässerige Decoct ist durchsichtig, braun, von vuangenehmem Geruche und sehr bitterm adstringirendem Geschmache. Galläpfeltinctur erzeugt einen reicblichen schmatrig-gelben Viederschlag, Weingeist macht die F'ar be heller, aber trübt zugleich auch etwas die Flüssigkeit, salzsaares Eisenoxyd bewirkt eine dunklere und lodtinctur eine blasgrüne Färbang.

Eine bei mäfsigem Feuer durch Digestion mit Weingeist bereitete Tinctur ist durchsichtig, gelbbraun, geruchlos, sehr bitter. Mit salzsturem Eisenoxyd färbt sicb die Tinctar dunkler, von Kohlensaurem Natron und Bleizucher entateht ein gelber Niederschlag und mit Gallëpfeltinctur nach einiger Zeit ein braungelber Präcipitat. - Das durcb Abrauchen der Tinctur erhaltene Extract ist trocken, braun, sebr bitter, in Wasser ganz löalich; diese Lösung ist braungelb, ond wird ron Galläpfeltinctur stark, ron Bleizucker aber nicht gotrubt.

Einem kleinen Hande wurde eine Dracbme der Warzel 
gegeben, es entstand aber nicht, wie man vermuthete, Erbrechen, noch konnte man sonst einen besondern Erfols wahrnehmen.

Von welcher Pfanze die Wurzel hommen möchte, ist darchaus ungewils.

Hr. Prof. Wenderoth in Marburg beschreibt ebenfalls (Pharm. Centralblatt 1836. No. 8. S. 113.) eine falsche Columbo auf folgende Art: Es ist das si Zoll starke Segment einer cylindrischen Wurzel (wahrscheinlich eines Menispermi) von beinahe a Zoll im Durchmesser. Rinden - und Holzkörper sind deutlich geschieden; aber nicht durch einen dunklern Kreis, wie bei der ächten Columbo, sondern lediglich durch die verschiedene Structur beider und ihre theilweise Trennung. Aeufserlich mit Oberhaut bedecht, ist diese runzlich, von unregelmälsigen Längsfurchen und Höckern; von Farbe grau-gelb-bräunlich, genau darin übereinstimmend mit einer andern falschen, die in Hleinen Scheibenstücken von dichterem Gewebe, innigem Zusammenhang beider Theile des exogenischen Pllanzenkörpers vorkommt, und fast geschmacklos ist. Die Rinde ist etwas über eine Linie dick, weifs, feinLờrnig, mit bräunlich gefärbten Strahlen der Rindenzellen. nie Farbe des Holzkürpers ist nach dem Umfange zu verschiedentlich gelb, selbst rothgelb gefleckt, nach innen weife. Man erkennt mit bewaffnetem Auge, wiewohl undeutlich, die concentrischen Kreise der Gefäfse (Jahrringe) und das sehr dichte Zellgewebe in der Form kleiner Poren. Die Substanz läfst sich leicht schneiden, und scheint sehr amylös. Sie ist specifisch schwerer als die ächte Columbo, die sich leicht an der gesättigten grünlichgelben Farbe, dem dunblern Kreis zwischen Rinden - und Holzkörper, den erhabenen Markstrahlen, welche mit jenem besonders nach dem Einweichen in Wasser deatlicher hervortreten, und den gleich im Anfang bei dem Hauen bemerkbaren rein bittern Geschmack unter- 
scheidet. Die falsche dagegen schmecht widerlich beifsend, welcher Geschmack relbst bei dem Kauen einer Weinon Menge (1 Gran) lange auf der Zunge und am Gaumen haftet.

\section{Officinelle Laurineen.}

Fast ron keiner andern Ptlanzenfamilie sind so viele Gewächse im medicinischen Gebrauche, als von der der Laurineen, aber auch nur wenige waren in botanischer Hinsicht so mangelhaft bekannt, als eben diese lorbeerartigen Gewächse, die mit wenigen Ausnahmen in den Tropenländern wobnend, bis jetzt nar sehr unvollatändig beschrieben warden, ja ron manchen Arznei-Drognen, welehe die Laarineen liefern, hannte man die Mutterplanzen ganz und gar nicht. Om so angenehmer ist es nun, endlich ein Werl 20 besitzen, in welchem diesem in der modicinisch-pharmaceutischen Botanik tief gefüblten Mangel grofsentheils abgeholfen worden ist. Wir rerdanken dasselbe (Syctema Laurinearum, Berolin. 1836) Herrn Professor Nees r. Esen beck dem Aelteren in Breslau, dem so viele, so bostbare und seltene Hülfsmittel zu Gebot standen, wie wohl kein anderer deutscher Botaniker sich rühmen kann, und der dieselben mit längat bekannter Omsicht und Scharfsinu so benutate, dafs diefs Werk einen bleibenden and klassischen Werth für die Naturgeschichte der Arzneipflanzen behalten wird. Es dürfte darum den Lesern der Annalen angenehm seyn, hier das zusammengestellt zu finden, was speciell zur Henntnifs der officinellen Laurineen gehört, wiewobl das ganze Werk vorzugswreise zum Studium benutzt 20 werden rerdient.

Hr. Prof. N. r. E. theilt die ganze Familie der Laurineen in folgende Stämme oder Tribus ein:

Tribus I. Cinnamomeae. Bermophroditae. Staminodia perfecta. Antherae 4 locellatae, interiores retroversae. Limbus 
perianthii articulatus. Gemmae incompletae. Folia perennantia, per paria approximata tri rel triplinervia.

Dieser Tribus begreift nu, die einzige Gattung Cinnamo. mum Bu r m a n n, deren Glieder sämmlich in Ostindien wachsen. Es gebören dahin uuter andern: Cinnamomum obtusifolium Noes and C. Bazania N., woron besonders die Rinde des letzteren nach Hamilton einen angenehmen Zimmt-Geruch and Geschmacls besitat. Cinnamomum Malabatbram Blame (nec Batka), woza die so viel besprochene Katou Raran des Rhoede gehört, und wovon eine scblechte Sorte malabarischen Zimmts stammt. Cinnamnmum camphoratam Blumo oder Laurus pseudn-Cassia Rein waldt, in Java einheimisch, wächst nur stranchartig und hat eine scharf aromatiscbe Rinde, die stark nach Muskatennufs riecht, mit einem deutlichen Beigeruche nach Camphor, welcher Geruch angenebmer an der trockenen, als an der frischen Rinde ist. Cinnamomum encalyptoides Nees, Laurus Cassia der botanischen Gärten (zum Theil), ansgezeichnet durch das Arom seiner Rinde und Blätter. Cinnamomum nitidum Nees oder Laurns nitida Rox. burgh, der rorigen Art verwandt, und auch mit einem, obgleich nicht starken, Zimmt-Geruch und Geschmack der Rinde verseben.

Cinnamomum zeylanicum Breyn, der zeylanische Zimmtbanm, von dem hier folgende Varietäten unterschieden werden: Commune foliis rel ovatis vel orato-oblongis obtusis rel brevissime obtusissimeque cuspidatis, cortice aromatico odore cinnamomeo. Davon gibt es eine ganz ähnliche Spielart, deren Ainde jedoch ganz gewürzlos ist, und eine andere bleinblättrige, deren Hinde hōchst angenehm aromatisch ist: $S$ ubcordatum mit fast berzförmigen stumpfen Blättern, Ha y ne Arzneipft. XIl. tab. 21. Cassia foliis oblongis vel ellipticis apice longins attenuatis basi acutis. Dahin gehort unter andern 
Laurus Caesia Lo, Cassia lignea Blach well tab. 3q1. Icon. exclus. Synon. u. s. w.

Cinnamomum aromaticum Nees oder Laurus Cassia $\mathrm{Ai}$ to $\mathrm{a}$, in China einheimisch and, wie man weifs, der Baum, ron welchem der allbekannte indische oder chinesische Zimmt kommt.

Cinnamomum sulphuratum Nees oder C. japanicum Blame. Die Bindo ist braun and hat einen schwachen Zimmt. geschmack.

Cinnamornum Capparu-Coronde Blume, in Zeylan einheimisch. Die Rinde soll ebedem für wahren zeylanischen Zimmt verkauft worden seyn, aber sie sieht viel dunkler roth ans, riecht nach Camphor und hat heineswegs das liebliche Arom des wahren zeylanischen Zimmtbaums.

Cinnamomum Tamala Nees, ausgezeichnet durch die gewürzhaften Blätter, die getrocknet anfangs nach Zimmt, und wenn man sie länger kaut, nelkenartig mit einem Beigeschmack nach Camphor schmecken; auch Cinnamomum Rauwolffi Blome hat Gewürzblätter, die in den Handel gebracht werden.

Cinnamomum albiflorum Nees oder Lauras Cassia Roxburgh and L. Soncaurium Hamilton hat nicht minder aromatische Blätter, die aber nicht so geachtet sind wie die von C. Tamala.

Cinnamomum Sintok Blume, dessen Ainde anch in Europa bekannt wurde: sie ist braun, schmeckt bitterlich and gewurrzhaft, wie ein Gemische aus Zimmt und Nelben.

Cinnamomum dulce Neos oder Co chinense Blame hat eine braune Rinde mit einem, jedoch nur äufserst schwachen, Zimmt-Geruch und Geschmack.

Cinnamomum Loureirii Nees oder Laurus Cinnamomum Louroiro, eine Art, die früher als Synonym zu C. aroma. 
ticum gezogen wurde and besonders darum interessant it, weil ron ihr die Flores Cassiae der Apotheken tsommen. Diese Nachricht stammt ron dem berühmten Reisenden Hrn. ron Siebold, der sie seinerseits einem ibm wohlbetannten Cbinesen verdantt, welcher sehr wohl anterrichtet za sejn schien. Die sebr dicken Aeste des Baums geben einen schlech. ten Zimmt, der meistentheils weggeworfen wird, da er die Kosten des Transportes nicht ersetat. Die dünnere, von den jüngeren Zweigen genommene Rinde gleicht an Dicho dem zeylanischen Zimmt, schmeckt und riecht aber sehr scharf, - ist darum nicht sehr geachtet, and nur die Cochinchinesen würzen ihre Speisen damit. Aber die Rinde der mittleren Aeste, die ungefähr 1 Linie dick ist, liefert einen sebr guten und hostbaren Zimmt, der zur Arznei dient, and selbst viel theurer verkauft wird, als der zeylanische, zumal da er in Tunkin wächst und selten gefunden wird. Durch Destillation liefert dieser Zimmt reichlich ein ätherisches Od, von rothbranner Farbe, das weniger scharf und viel süfser ist, als das vom zeylanischen Zimmt.

Cinnamomum Kiamis N'ees liefert eine Sorte des Cortex Massoi der Officinen; diese Rinde ist dick, braun, von eigenthümlichem Geruch und süfslich adstringirendem Geschmach, an die Alyria erinnernd, und etwas schleimig. Cinnamomum paucifloram Nees oder Laurus albiflora Wallich hat eine der vorigen ziemlich ähnliche Rinde. Auch Cinnamomum Reinwardtii Nees oder Laurus Culilawan Reinw. hat eine braune, schleimige, nach Nelken und Zimmt riechende und schmeckende Rinde, die nicht minder mit Cortex Mascoi manche Aehnlichleit hat.

Cinnamomum Culilawan Nees liefert, so wie C. robrum Bl ume, die. Nelkenrinde, worüber nächstens einige Notizen folgen werden.

Cinnamomum xanthoiseurum Blume liefert den Cortex 
Culilawan Papnanum, der der im malajischen Archipel aberall Massoi genannten Rinde sehr ahnlich ist.

Noch ist Cinnamomum vimineum Nees zn erwähnen, dessen blafabraune Binde fenchelartig wie Sassafras riecht, übrigens hat der Banm nur zweifelhaft seine Stelle in dieser Gattung erhalten.

Tribus II. Camphoreae. Hermaphroditae. Staminodia perfecta. Antherao quadrilocellatae, interiores retrorersae. Limbus perianthii decidaus. Gemmae perulatae. Folie perennantia longe petiolata, triplinervia ant subtriplinervia, in axillis costarum glandulose subtusque porosa.

Es sind asiatische Bäume, zumal in China und Japan einbeimisch, die nur die einzige Gattung Camphora Banhini ausmachen. - Allbelannt ist Camphora officinarum Bauk. oder Laurus Camphora L., wozu noch einige rerwandte Arten Lommen, wie Camphore inuncta Nees und C. chinensis Nees, die beide vielleicht nar Varietäten oder Gartenformen der C. officinarum sind; sodann Camphora glandulifera Nees oder Lanrus glandulifera $\mathbf{W a l l i c h}$, in Nepal einheimisch; ein hoher Banm mit brauner, nach Camphor riecheader und achmeckender Biade.

Tribus III. Phoebeace Hermapbroditae. Staminodia perfecta. Antherae bi - rel quadrilocellatae, interiores retroversee. Limbus perianthii persistens, durescens, cupulam bacese adpressam strueno. Gemmae incompletae. Folia perennantia venosa et penninervia.

Es sind Bäume, die auf den hanarischen Insela, 80 wio in Ost - and Westindien wachsen; sie bilden 2 Gattungen: Apollonias (antheris bilocellatis) and Phoebe (Ocotea anctoram, antheris quadrilocellatis).

Zum medicinischen Gebrauche scheinen die zahlreichen Arten dieser Abtheilung wenig verwendet worden zu sejn; 
weaigstens ist deren Rinde grofeentheils geruchlos und allenfalle adstringirend.

Tribus IV. Perseae. Hermaphroditas ant rarins diclines. Staminodia perfecta. Antherae bi-quadrilocellatae, interiores retroversae. Limbus perianthii rol persistens, et tum sob fructu patulus non mutatus, nec lignescens, rel deciduus discum orbicularem relinquens. Pedicelli fructus in plurimis incrassati, succelenti vel subcarnosi. Gemmae incompletse. Folia perennantia, penninervia saepe paberala. Florum paniculae amplae, vel ubi minores sunt rigidulae, cymulosae, plerisque infratermioales. Perianthia grandiuscala, pube restita.

Die meisten Bäume dieser $\mathbf{A b t h e i l u n g ~ s i n d ~ i m ~ t r o p i s c h e n ~}$ Amerika einheimisch, einer oder der andere im nördlichen Amerika oder den kanarischen Inseln, einige in Ostindien. Eine ganze Reihe derselben gehört den Alpen-Gegenden der Tropen im neuen Continente. Diese Abtheilung umschliefst fünf Gattungen: Persea, Machilus, Baldu, Alseodaphne, Hufelandia. - Es gehören dahin unter andern: Persea gratissima Gärtner, deren Frucht in heifsen Liändern zu den geschätztesten Obstarten gezählt wird; Persea indica Sprengel, deren Hoľ an Schönheit und Dauer dem der Swietenia Mabagoni nicht nachstehen soll; Persea (Eriodaphne) pyrifolia Nees, ein brasilischer Baum, hat eine braune, etwas nelkenartig riechende Minde und einen eigenen, an die Schärfe der 8arsaparilla erinnernden Geschmack; Persea (Eriodaphne) carolinensis N. oder Laurus Borbonia L., in den dichten Cypressen-Wäldern Virginiens und in Lonisiana einheimisch, hat ein vortreffliches Holz, die Rinde, so wie fast alle Theile des Baums sind aromatisch. Machilus odoratissima Nees aus Nepal, durch den herrlichen Geruch der Blumen ausgezeich. net. Baldu chilanum Nees aus Chili, ein boher Baum mit dünner, brüunlicher, aromatischer Rinde; in Chili räuchert man die Fässer damit aus, ehe Wein in diese gebracht wird; 
nach Grabam ist diese Binde zimmtartig, auch dient ein Decoct derselben in Bädern gegen Syphilis, Wassersucht und rheumatische Leiden. Endlich ist noch Alseodaphne cymbarum Kosteletzky zu erwähnen, ein am Orinoco einbeimischer Baum, mit sehr bilterer, wohlriechender Rinde. Man glaubt, dafs das in der nevesten Zeit bekannt gewordene, natürliche Lorbeeröl, welches man im columbischen Gujana durch Einschnitte in die Rinde gewinnt, von diesem Banme abstamme.

Tribus V. Cryptocaryeae. Hermaphroditae. Staminodia perfecta (quibusdam nullo?). Antberae bi - rarius quadrilocellatae, locellis faeiei insertis, interiores retroversae. Limbos perianthii deciduus vel persistens. Namerus stamiaum in quibusdam abortu diminutus. Fructus vel siccus vel baccatas, in perianthii tubo vel baccante vel durescente totus maximamve partem inclusus, rarissime perianthii basi persistenti impositus ct tanc vero durus nec baccatus. Inflorescentia vel thyrsoidea densiuscula, vel racemosa. Perianthium plerisque infundibuliforme. Gemmae incompletae. Folia perennantia, renosa, paucic nervosa.

Es sind ostindische oder neuholländische Bäume, nur wenige sind im tropischen Amerika einheimisch. Es gehören dahin die Gattangen: Endiandra, Beilschmiedia, Cecicodaphne, Cryptocarya, Caryodaphne, Agatophyllum, Mespilodaphne.

Ansgezeichnet ist die Rinde der Beilschmiedia fagifolia Nees durch ihren scharfen aromatischen Piunent-Geschmack; Cecicodaphne glaucescens hat eine dünne bräunliche Rinde mit Myrrhen-Geschmach; Cryptocarya moschata Martius oder Ocotea cymbarum $\mathrm{Humb}$. hat eine sehr angenehme arumatische, wie Piment and Nelken riechende and schmectuende Rinde, welche Eigenschaften dio Frucht noch ausgezeichneter besitzt. Cryptocarya forrea Blume bat, wie schon der Name 
errathen lärst, ein äufserst festes, dauerhaftes, aber schwer zu bearbeitendea Holz. Cryptocarya Peumus Nees, in Chili einheimisch, hat eine dünne, eigenthümlich camphorartig riochende Rinde. Caryodaphne densiflora Blame, in Java einbeimisch, liefert eine röthliche, bittere, balsamisehe Rinde, die als ein tonisches Mittel im Gebrauche ist. Die angenehm aromatischen Blätter werden als Thee bei Magenkrämpfen und Convulsionen der Schwangern gebraucht.

Agatophyllum aromaticum Willd. hat eive aromatische Frucht, die unter dem Namen Nelkennufs, Ravensara, oder Quatre èpices auch in Deutschland in den Handel ham; auch die Hinde hat einen starken angenehmen nelkeartigen Geruch. Die Blätter wurden bei der Versammlung der Naturforscher und Aerzte in Stuttgart von Jobst vorgelegt.

Mespllodaphne pretiosa $N$. et M. oder Laurus Quixos Lamark, in Brasilien einheimisch, ist ein höchst ausgezeichneter Gewürabaum, deasen innere Rindenlagen ein glënzeades Anseben haben und einen höchst angenebmen Geruch and Geschmack besitzen, den man mit einem Gemische aus Zimmt, Pomeranzenblüthen und Bergamottöl verglichen hat; auch die Kelchrinde zeigt dieses liebliche $\Delta$ rom. Es ist diefs die Canelilla oder der berühmte Zimmt rom Orinoco nach v. H u mboldt. Mespilodaphne leucophlaea hat ebenfalls eine avgenehme, doch schwächer aromatische Rinde, was anch ron M. prolifera Nees zu sagen ist.

Tribus VI. Acrodiclidia. Arbores hermaphroditae. Staminodia rel nulla, vel dentiformia compressa. Stamina tria vel novem. Antherae locellis binis poriformibus, juxta opicem dehiscentes, subsessiles, interiores retro aut introversae. Fructus primum perianthio pomiformi vel mespiliformi laciniis conniventibus clauso aut umbonato reconditus, serius emergens et cupulae calycinae crassae truncatae vel appendiculatao profunde inmersus, baccans. Inflorescentia paniculata thyp- 
soidea, rel conglomerato-capitata puberula. Flores saepe infundibuliformes limbo brevi. Gemmae incompletae. Folia perennantia, venosa, cuspidata, saepius puberula.

Es sind im tropischen Amerika einheimische Bäume, bo. stehend aus den Gattungen Aydendron, Eronymodaphne, Acrodiclidium und Misantera.

Besonders zu erwähnen ist Aydendron permolle Nees, mit brauner, glänzender, harziger Rinde und einem harten, grauen, angenehm wie Bergamottöl riechenden Holze. Von Aydendron Cujumary Nees sind die Saamen aromatisch und enthalten dabei selbst noch mehr einer fetten Substanz als die bekannten Pichurimbohnen. Man braucht sie wie diese bei Verdaunngabeschwerden, in Pulverform mit Wasser oder Wein gemiecht; gröfsere Wirksamkeit für Magenkrankheiten schreibt man noch einer Mischung zu aus gleichen Theilea des Pulvers dieser Früchte und dem halbverbrannten Holze eines systematisch noch nicht bestimmten Baumes, Pira-cuaba, d. h. Fischholz genannt.

Aydendron Laurel Nees, Ocotea Pichurim Humb. oder Laurus Pichurim Willd. hat ein angenehm riechendes Holz, and seine Früchte sind in jeder Rücksicht den Pichurimbobnen nahe verwandt, und mehr noch jenen des Cujumary.

Aydendron firmulam Nees hat eine dicke, braune, etwas bittere Rinde mit einem aromatisclien Geruch und Geschmack nach Cardamomen; auch Aydendron salicifolium Nees hat eine aromatische Rinde, eben so $\mathbf{A}$. microbotryum N., $\Delta$. suareolens N. u. s. w. Misantera capitate hat eine brïabliche, scharf nach Pfeffer schmeckende and riechende Rinde.

Tribus VII. Nectandreac. Hermaphroditae. Staminodia dentiformia, rarius subcapitata. Stamina norem. Antherso subsessiles, latae, locellis banin rersus in arca dispositis, dehiscentes, interiores retroverase. Periantbii laciniae latiusculae, patentes, deciduas, exteriores latiores. Fructus baccatus co. 
palae profundae truncatae insertus. Inflorescentia: panicula, in plerieque ampla. Gemmae incompletae. Folia perenoantia, penninervia, venosa, in axillis venarum costalium quabdoque poroso.

Es sind Bäume des tropischen Amerika, nur pine einzige Gattung, Nectandra, bildend, die jedoch in zwei Sectionen • zerfällt: Pomatia, staminodiis biglandulosis, und Porostema, staminodiis nudis. Unter den zablreichen Arten dieser $\mathbf{A b}$. theilung mafs man besonders auf die nachstehenden aufmerksam macben:

Nectandra cymbarum Nees, Sassafras rom Orinoco, Ocotea cymbarnm $\mathbf{H}$ o mb. oder Ocotea amara Mart., mit etwas dicher, brauner, aromatisch-bitterer Rinde, die in Brasilien als $\mathbf{H i s -}$ genmittel dient, and nach Martias zum Urari-Gift gemischt prerden soll.

Neclandra cinnamomoides Nees, Lauras cinnamomoides Mutis, Canella do Mato, wilder amerikanischer Zimmt; doch wird der Baum auch cultivirt, wahrscbeinlich wegen seiner distinct nach Zimmt riechenden und schmeckenden Ainde.

Nectandra (Porostema) Puchury major N. et M., der wabre Baum, ron dem die grofsen Pichurimbohaen der Apotheluen herrührea. Nectandra Puchury minor N. et M. liefert die Heineren Bohnen dieses Namens; allein es ist zweifelhaft, ob der Banm wirklich zur Gattung Nectandra gehört, da dio Blamea unbekannt sind.

Tribus VIII. Dicypellia. Dioica vel polygama. Staminodia nulla Stamina ordinum duorum exteriorum in foemina lacinits limbi consimilia et cum iisdem persistentia calyculum crassum radiatum sub fructu formantia, vel cadentia (?). Antherae interiures 3-6 sessiles, quadriporosa. Bacoa. Inflorescentia racemosa depauperata, saepe bracteolata et ab initio gemmiformis. Gemmae incompletae. Folia perennantia renose

Es sind brasilische Bäume, die noch nioht zareichend 
bolsant aind, und daber später noch andern Sectionen zagetheilt werden hönnen. Besonders interessant ist Dicypellium caryophyllatum Nees, wovon die brasilische Nelkenrindo abutammt. Noch gehören in diese Abtheilung die Gattungen Petalanthera und Pleurothryum.

Tribus IX. Oreodaphneae. Dioicae vel polygamae. Staminodia nulla vel subulata, ant saltem imperfecta. Stamina vel 6, tribus interioribus deficientibus, vel in plerisque 9 , rarius 12, tertii (quarti etiam, si adest) ordinis reversa. Antherae filamento distincte praeditae, $2-4$ locellatae, abi 4 locellatae, locelli per paria sibi sunt impositi. Perianthii campanulati vel rotati, parvi vel mediocris, rarissime grandioris laciniae aequales nec dilatatae. Fructus parrus vel mediocris, baccans ant cupulae profundae truncatae appendiculataeve insertas, ant perianthio patulo immutato suffultus, aut pedicelio fere nudo incrassatoque subcarnoso impositus. - Inflorescentia axillaris rel infraterminalis, paniculata vel racemosa, multis parra et racemulosa, quibusdam tenera et laxa aut elongata, minuti bracteolata, bracteolis fugacibus: in una specie umbellulata et inrolucrata. - Gemmae incompletae. Folia perennantia venosa, multis in axillis venarum costalium subtus porose et barbalata, saepe salicina, tam Caprearum quum Vimsinalium folits comparanda.

Es sind Bäume und Strüucher des tropischen Amoriks, wenige wachsen auf den Mascarenhas, eine einzige auf den Lanarischen Inseln; es gehören dahin die Gattungen: Telejandra, Leptodaphne, Ajouea, Göppertia, Dehaasia, Oreadaphne, Camphoromoea, Ocotea und Gymnobalanus.

Unter den zahlreichen Arten mŏgen bier nur eine Stelle finden: Hassia elongata Blume, ein hober Baum mit branver, ranzlicher, aromatischer, bitterer Rinde. Oreodaphne opifera N. et Mart., aus dessen Früchten dorch Destillation ein Llares ätherisches Oel erhalten wird, von gelber Wein- 
farbe, wie ein Gemische von Citronen- und Rosmarinöl riechend und scharf aromatisch schmeckend. Man bedient sich desselben in Brasilien bei Contracturen der Glieder, bei rbeumatischen Beschwerden u. s. w., wo es eatweder für sich, oder mit einem fetten Oele zur Salbe gemiscbt, eingerieben wird. Oreodaphne exaltata Nees liefert ein gans vorzügliches hartes, so lange es frisch ist, gelbes Ziminerholz. Oreodaphne indecora Nees hat eine braune, nicht unangenehm riechende, scherf und bitterlich schmeckende Finde; wie denn überhaupt noch mehrere andere Arten dieser Gattung ein eigenes Arom besitzen. Von Oreodaphne foetens Nees sagt Herr v. Buch: Es ist einer der grṓsten Bäume anf den Lenarischen Inseln, den aber keine Axt ungestraft berührt oder rerwundet. Der sich entwickelnde Gestank aus dem Holze ist so heftig, dals er die Arbeiter zur Flucht zwingt, 20 dafs sje einen Baum nur nach langen Unterbrechnngen in mehreren Tagen zu fällen im Stande sind. Die Rinde ron Oreodaphne maximiliana $\mathrm{Nees}$ et $\mathrm{M}$ art. riecht und schmecht dagegen stork and angenehm nach Nelten.

Tribus X. Flaviflorae. Dioicae vel polygamae. Staminodia nulla. Stamina novem. Antherae $2-4$ locellatae, omnes introrsum dehiscentes. Perianthiun rotatom, tenue, flavum. Bacca pedicello subnudo quandoque incrassato imposita. Racemuli vel Umbellulae praecoces, involucratae aat bracteatae. Gemmae perulatae. Folia membranacea decidna.

Es sind Bäume und Sträucher des nördlichen Amerika und der nepalischen Alpen, bestehend aus den Gattungen Sassafras und Benzoin.

Wir haben hier zu nennen Sassafras officinalis Noes, wovon das gemeine oder rothe Sassafrabholz, and Sassafras albidum Nees, woron das weifse oder glatte Sassafrasholz bommt, und wahrsebeinlich mit dem rothen gemiscbt, auch zu uns gebracht wird. Sassafras Parthenoxylon Nees, in 
Sumatra und Java einheimisch, hat ein weiches, poröses, röthliches Holz ron angenehm aromatischem. süfslichem SassatrasGeschmacke. Dic Blumen riechen zerrieben stark nach Camphor; auch wurde der Baum früher in die Gattong Camphora gerechnet. Blume nannte ihn Laurus pseudo-Sassafras.

Arten von Benzoin sind vier aufgeführt, nämlich B. melissifolium Necs, B. aestivale N. oder Laurus Benzoin Willdenow, die bei uns in den Gärten gemeinste Art; dann $B$. odoriferum Nees oder Laurus Benzoin Linnaei; endlich Benzoin Neesianum Nees aus Nopal, dessen schwarze Beeren stark nach Oleum Cajeput riechen.

Kosteletzly benut für diese Plianzen den GattungsNamen Benzoin nicht an, sondern nennt das Genus nach Presl in Prag Calosmon; er sagt: Diese Gattung bat zwar von den neuesten Monographen den Namen Benzoin erhalten, der jodoch aus doppelten Gründen nicht angenommen węrden darf, da erstens achon eine Gattung dieses Namens besteht, und zweitens gegenwärtige Gattung bereits vor mehreren Jahren. unter dem Namen Calosmon beschrieben wurde.

Tribus XI. Tetranthereae. Dioicae. Staminodia nulla. Stamina 9-18 plerisque longa; ubi plura quam novem, perianthium limbo vel caret pel partium numero diminuitur. Antherae 2 rel 4 locellatae, cunctae in plurimis introrsum dehiscentes, in uno genere (Cylicodaphne) interioris ordinis antherae extrorsum. Bacca tubo perianthii patelliformi vel explanato imposita. Inflorescentia : aut umbellulae involucratac aut fasciculi primum squamis gemmae unifloris ant paucifloris perulati. Gemmae incompletae, rel superiores tantum squamis 4-6 exterioribus praeditae. Folia renosa plerisque perennia.

Es sind meistentheils ostindische Bäume, nur wenige wachsen im gemälsigten Amerika, eine einzige in Europa. Es gehören dahin die Gattungen: Cylicodaphne, Tetrantbera, 
Polyadenia, Laurus, und Lepidadenia. Aufser dem gemeinen Lorbeerbanm scheinen wenige Arten dieser Section im medicinischen Gebrauche zu seyn, wenn es gleich nicht an aro. matischen und andern heilträftigen Bestandtheilen mangelt, wie namentlich Totranthera glaucescens 3 prengel in allen ihren 'Theilen gewürzhaft ist.

Tribus XII. Daphnidinae. Dioicae vel hermaphroditae. Staminodia nulla. Stamian 9-19, et ubi plora quam partes perianthii, istae non deficiunt. Antherae $2-4$ locellatae, omnes introrsum dehiscentel. Bacce tubo perianthii vel discoideo vel cyathiformi imposita, vel pedicello nudo. Gemmae peralatae. Flores e gemma geniti, squamis bracteati, axillari-sob. glomerati. Folia perennia, aliis renosa, aliis nerrosa.

Es sind Bäume, die in Ostindien, China und Japan einbeimisch sind, enthaltend die Gattungen Dodecadenia, Actinodaphne, Daphnidium und Litsaea. Zu erwähnen sind besonders: Daphnidium Myrrba Nees, dessen Wurzel braun und ölig, die ganze Pflanze sebr bitter ist and ganz den Gerach und Geschmach der officinellen Myrrhe besitzt. Daphnidium gracile Nees, dessen Vaterland anbehannt ist, wird in mehreren botanischen Gärten Deutschlands unter dem Namen Laurus Culilawan cultivirt, hat eine aromatisch myrrhenartige Rinde und blüht in den warmen Häusern im Februar oder März; anch unter den Namen Laurus glanca und $L$ gracilis findet sich diefs Bäumchon in den Treibhäusern; noch Hommt in diesen unter dem Namen Laurus Culitlawan ein zweites Bänmchen vor, ebenfalls unbekannter Herkunft, das Herr Prof. Nees nun Litsaea Pseudoculitlawan nennt. - Daphnidium Cubeba Nees hat eine Frucht, die der Form and den Eigenschaften nach mit denen von Piper Cubeba übereinsommt, and in Cochinchina nicht nur als Gewürz, sondern auch als Arzneimittel benutzt wird. Von Littaea consimilis Nees riechen alle Theile, zumal Wurzel und Beeren, nach 
Camphor, und der Baum selbst hat das Ansehen des Lauras Camphora L.

Tribus XIII. Cussyteae. Hermaphroditae. Staminodia perfecta. Stamina norem, antheris quadrilucollatis, tribus interioribus reversis. Caryopsis perianthio baccato laciniis conniventibus coronato inclusa. Folia nulla.

Es sind ostindische Schmarotzerpflanzen, die das Ansehen unserer deutschen Arten von Cuscuta haben; sie kommen auch in Neubolland, so wie in dem wärmeren Afrike und Amerika vor, und bilden nur die einzige Gatung Cassyta. Am be. kanntesten ist Cassyta Gliformis L, welche Pllanze nach R $\mathbf{m} \mathbf{p h}$ voll von einem hlebrigen Schleime ist, wefshalb die Javaner und Malajer dis ganze Pflanze zu einem Brei zerstofsen, mit Kalkpulver mischen und damit ihre Kähne überziehen. Die jungen zerstorsenen Triebe übergiefot man mit Buttermilch, die davon dick wird, und die man Fieberkranken als ein kühlendes Mittel zu trinken gibt. Auch soll die Klebrigkeit der Cassyta, äufsurlich angewendet, den Haarwachs befördern.

Noch fübrt Herr Prof. N. mehrere Pllanzen an, die man in die Gattung Laurus zählte, die dahin nicht, und mancho nicht einmal in die Familie der Laurineen gehören, wie Laurus umbellata Thanberg, Laurus caustica Molina. - Lauras Sassafras Loureiro, Laurus joritensis $H$. et $K$. a. m. a. Honnten nicht mit Sicherheit in eine bestimmte Section ge- $^{6}$ bracht werden.

Man vermifst auch Lanrus sanguinea $\mathrm{S}$ wartz, welchen Baum Herr Prof. Hostele tz ky zu der Gattung Cylicodaphne rechnet, und davon eagt, er liefere im Innern von Brasilien eine Zimmtrinde, die daselbst unter dem Namen MaragnanZimmt belsennt, und einen nicht unbeträchtiichen Handelsartibel für die Indianer am Imazonenstrome ausmache.

Noch beschroibt Herr Prof. N. die Familie der Illigereas 
Blume, die er früher Laurinae Gyrocarpae nannte; begreift nur wenige Arten, die bald zu den Myrobalaneen, bald zu den Thymelaeen, bald zu den Combretaceen gerechnet warden, und jetzt zwei Gattungen, Illigera und Gyrocarpus, ausmachen; es sind tropische Bäume, die in Asien, Neaholland und Amerika wachsen.

\section{Radix Serpentariae.}

Eine sehr ausführliche Beschreibung der Pflanze oder Pflanzen, welche dieses bekannte Arzneimittel liefern, hat Rafinesque in seiner Beschreibung der in den rereinigten Stauten ron Nord-Amerika gebräuchlichen Arzneipflanzen gegeben, welches in Philadelphia heraus ham und das Ref. leider nicht sab. Nor von diesem Artilel bat die Linnaea (Bd. q. Literat. S. 95.) einen vollständigen Auszug gegeben, woher Folgendes entrommen ist.

Aristolochia Serpentaria: Stengel einfach, vielbeugig; Blätter lanzettlich, herzförmig, ganz und zugespitzt; Blumen 2-lippig, fast wurzelständig; Blumenstiele gekrümmt, einblumig, schuppig, gegliedert.

Beschreibung. - Wurzel ausdauernd, knotig und böckerig, braun und sehr zaserig, die Zasern lang, dünn, frisch gelb. Stengel rund, schlank, schwach, vielbeugig, gegliedert, weniger als einen Fuf́s hoch, 1-3 Blumen. Blätter wechseind and gestielt, länglich oder lanzettlich, mit bleiner herzformiger Basis und zugespitzter Spitze, ganzem, zuweilen welligem Rande, kahler oder weichhaariger blafsgrüner OberAläche. Blumen fast wurzelständig und einzeln aufgebogenen gegliederten, farbigen, mit einigen Heinen Schuppen besetzten Blumenstielen. Fruchtknoten unterständig, Perigon röthlich oder purpurröthlich, Röhre krumm, Band a-lippig, Oberlippe gekerbt, Unterlippe ganz, beide turz und gelappt. Sechs sitzende Staubbeutel sind an den Seiten der breiten, runden, 
aitzenden Narbe beleatigt. Kapsel umgekehrt-eiförmig, mit 6 Fächern and vielen tleinen Saamen.

Geschichte - Die Gattang Aristolochia fordert eine durch. gängige Untersuchung und Verbesserung, da sie eher eine Familie, als eine Gattung ist; 2wei Untergattungen müssen wenigstens ron ihr gebildet werden: 1) Glossula, Blumen einlippig and bandformig. Wahrer Typus der Gattung. 2) $p_{i-}$ stolochia, Blumen 2-lippig and rachenförmig. Dazu gehören A. sorpentaria, A. ringens, A. bilabiata u. 8. w. Da viele Arten von den Gattungscharaltteren weit abweichen, müsten sie besondere Gattungen bilden, so: Siphisia, Blumen nicht lippenförmig, Hand gleich, 3-lappig; dergleichen sind: $A$. Sipho, A. tripteris, A. tomentosa u. s. w. Endodoca, mit 12 Staubgefäsen, 2. B. dodecandra and vielleicht Bigelow's A. Serpentaria. Einomeja, nur mit 5 Staubgefäfren und 5 . fächriger Kapsel, solche ist A. pentandra u. a.

Die in Rede stebende Art ist bis jetzt durchaus nicht gehörig begrenzt. Die virginische Schlangenwurzel des Handels wird ron einem halken Dutzend Arten oder Abarten gesammelt, A. hastata, A. tomentosa und mancher anderen, welche A. Serpentaria genannt werden, weil sio ähnlicho Blätter und Wurzeln haben, während die Blumen verschieden sind. Dio A. Serpentaria von Barlo n scheint eine besondere Abänderung mit langen schlanken Blumenstielen, relche wenig Schuppen haben und nicht gefärbt sind, wäbrend die Blamen blein, purparn und kaum 2-lippig sind. Bigelow's Pflanze, welche aus den südlichen Staaten ist, bat 3-nervige Blätter, welche weniger zugespitzt, aber. mehr wellig sind, während die Blumen grölser, 2-lippig und roth sind, viele and breite Schuppen, 12 Staubgefälse! und eingelappte zusammengerollte Narben. Diefs mag eine besondere Art seyn, welche vielleicht $\mathrm{za}$ Endodeca gehört. Unsere Abbildung ist von eiser grofsblumigen Varietüt der westlichen Waldblörsen, 
aber viele andere Varietäten gibt es hier, eine hat breite Blätter.

Allo diese Pflanzen blühen selton oder einmal in ihnom Leben im Mai oder Juni. Da sie, mit Ausnahmo der A. to mentosa, einander sehr ähnlich sind, so werden sie ohne Unterachied gesammelt. Die Warzeln allein hommen in den Handel und werden thenrer verkant, als die Benegaschlangonwurzel. Sie sind ein Ausfahrartikel- nach Europa.

Aristolochia gehört mit Asarum zor natürlichen Ordnong der Asarideae. Linnæus hat sie in die Gynandria hexandria gestellt

Standort: In schattigen Wäldern von Neu-England bis Florida and Missuri, sehr kräftig in den Allegbany - and Cumberland.Gebirgen, selten in den angeschwemmten und Kalls - Gegenden.

Wir übergehen bier die Eigenschaften and Wirkungen dieses Mittels, welche nun folgen, und gehen au den beiden letaten Abschnitten dieses Artikels über.

Ersatzmiltel. - Campbor, Rosmarin, Senega, Eupatoriam perfoliatam, Asarum Canadense und Virginicum, alle einheimischen Aristolochien, Gaultheria procombens und viele andere tonisohe und diaphoretische Reizmittel.

Bemerkungen: Die Rinde, Saamen und Wurzel der A. Sipho (oder Siphisia glabra), gemeinhin Holländer. Pfeifen. Blume oder Pfeifen-Wein genannt, hönnen substituirt worden, da sie dieselben Eigenschaften haben. Es ist oin hohes Klimmgewächs mit grofsen herzformigen kahles Blättern, und braunen, einer Pfeife ähnlichen Blumen, mit dreilappiger Oeffunog, welches am Obio wächst u. s. w. A. tomentase (oder Siphisia tomentosa) ist eine niedrige Klimmptanze mit herzformigen wolligen Blättern, welche in den weșlichen Staaten wächst. A. hastata ist eine kleine Pffanze mit lingen schmalen Blättern, die am Grunde stumpfe Oohrcben baben; 
sie wäobst in den südllchen Staateo. Die Wurzeln dieser beiden letzten werden oft mit denen der gemeinen Art in den Handlungen gemengt.

Henry's Figur stelit wahrscheinlioh dio A. tomentosa vor, aber die Blätter aind zu scharf.

Hier möge auch noch die Bemerhung stehen, daft nach Schrader mit der Wurzel der Aristolochia Bipho die Sarsaparilla verfïlscht in Handel rarkommt, welcher Betrug doch leicht zu entdection ist, denn die. Wurzel der A. Sipho riecht camphorartig, und die Fasern aind stärker als die der wahren Sarsa. - Noch eine zweite falscbe Sarsaparill entdeckto Stromeyer in Göttingen; sie stammat von einer unbehannten Planze, ist leichter als die wahre, von schmutzig weifser Farbe und ohne bemertlichen Gescbmack. (Man sebe Grttinger gelehrte Anzeigen 1834. 3. S. 1512.)

B. Gewächse aus der Abtheilung der Endogenen oder Monocotyledonen.

Veratrum viride Aiton.

Eine in Nordamerika einheimische Pflanze, deren Wurzel in den vereinigten Staaten unter dem Namen Radix Hellebori albi in den Apotheken aufbewahrt wird. Die Pilanze ist dem deutschen Veratrum Lobelianum Bernhardi nabe verwandt, und bat wie dieses grüne Blümchen, aber an der dentschen Planze ist die Hispe einfach und die Kelche glatt, bei der amerikanischen die Rispe zusammengesetzt und die Kelche gewimpert.

Dr. Ch. Osgood fand das Veratram viride in seinen Wirkangen eicherer und unschädlicher als das Veratrum albam und das Calchicum autamnale, indem die Gabe, in welcher es wirkt, sich sicherer bestimmen lasse, es auch kein unaufhörliches Abführen errege, noch jenen gereizten Zustand im 
Darmkanale zurücklasse, gleich dem Veratrum album. Es wirkt, den Beobachtungen des amerikanischen Prof. Fully zufolge, eröffnend, Brechen erregend, blasenziehend and scharf narkotisch. In voller Gabe (4-6 Gran in Substanz) mindert es auffallend die Stärke und Häufigkeit des Palses. Verf, rühmt es besonders gegen gichtische Entziindung, wo er Extract. Veratri viridis zu 1/3 Gran oder die Tinctur zn $1 / 2$ Drachme alle $3-4$ Stunden gibt, in allmäblig steigender Dosis, bis Narkose oder Brechen erfolgt. Zu gröfserer Sicher. heit empfiehlt er einen mälsigen Zusatz von Opium. Tinctura vinosa Veratri viridis $3 / a$ mit Tinct. Opii $1 / 4$ sollen in der Wirkung der Eau medicinale gleichhommen, nur dafs dieses Purgiren erregt. Gleich wirksam soll das Voratrum viride seyn gegen acuten Rheumatismus, wo es nur der Actaea racemosa nachstehe. F. gab hicr, nacb rocausgeschickten Gaben ron Calomel, eine Drachme 'f'inctara Veratri viridis mit Opium. In voller Gabe in kurzen Zwischenräumen gegeben, fand man es auch gegen die Pneumonia vera und notha sehr wirksam. Als Gegenmittel gegen die etwaige zu starke narkotische Wirkung empfiehlt der Verf. L!eine Gaben ron Laudanum mit Branntwein. Von den Präparaten dieses Mittels ist bis jetzt nur das Unguentum Veratri viridis in der nordameriBanischen Pharmacopoe als officinell aufgeführt. (American Journal Ang. 1835; Schmidt Jabrb. Bd. 11. Heft 1. S. 17.)

\section{Scilla maritima $\mathbf{L}$.}

Herr Ad. Stein heil, dem man schon mehrere schätzbare Abhandlangen über die Gewächse des nördlichen Afrika, zamal der Barbarei, verdankt, beschäftigte sich insbesondere aach mit den dort einheimischen Liliaceen, deren genaue Anordung in bestimmte Gattungen von jeher mit grofsen Schwierigheiten verbunden war. Bei dem Stadium einer aufgefundenen, wenig bekannten Liliacea jener Gegenden fand 
Herr 8. sich in die Nothwendigkeit gesetzt, eine neue Gattung anizustellen und bemerkte dann bald, dals in dieses neuo Genus manche Arten von Scilla, Ornithogalum, Albuca, Anthericum und Phalangium gebracht werden mufsten. *) Schon Mönch, sagt Herr S., habe den Scillen den Namen Stellaris gegeben, aber sie so definirt, dals sie mit den neueren Untersuchungen nicht in Einulang gebracht werden hann; Herr S. wäblte daram den Namen Urgirea, entnommen ron einem arabischen Stamme, der, in dem Territorium ron Bona sich herumtreibend, diese Benennung führt, in welcher Gegend die Planze gefunden wurde, welche die Veranlassung zur Aufstellung der neuen Gattung gab, die folgende Charaktere besitzt:

\section{Urginea.}

Calix coloratus, patens, sopalis tribus. Corolla calici fere similis; petala sepalis paulo latiora. Stamina sex, petalis breviora, filamentis glabris, basi subdilatatis, acuminatis, integerrimis, antheris muticis, oblongis. Ovarium tripartitum glabrum, Stylus glaber simplex, corolla brevior. Stigma subtrilobum papillosum. Pericarpium triloculare, trivalre, valvis medio septiferis. Semina plura, biserialia, complanato, testa membranacea, residactu laterali.

Es gehören in diese neue Gattung: Urginea fugax (Anthericum Moris.), $U$. anthericoides (Scilla Poiret), U.

-) Dieses Verfahren kann Ref. auf keinerlei Art billigen; der Name Scilla, der primitiv und seit Jahrtausenden sich an die Meerswiebel knüpt, murs ihr bleiben, wenn anders die Geschichte der Botanik und Medicin noch etwas gelten sollen. Das Verfahren des Herrn S., wenn es je Nachahmer finden sollte, würde die Syuonymie auf unnöthige Weise rermehren, und das Studium der Schriften der älteren Aerate und Botaniker sehr erschweren. 
fragrans (Anthericum Jicquin), U. filitolia (Anthericum Jacquin), U. exuviata (Anthericum Jacquin), U. undulata (Scilla Desfontaines) und

\section{Urginea Scilla Steinheil.}

Scilla sire Caepa marina Lo bel Hist. p. 75.

Scilla hispan. (Flor. et Sem.) Clus. rar, plant. histor. Lib. 2.

p. 171 .

Scilla magna rufa rulgaris Baub. hist. plant. t. 2. p. 6,5. Scilla Dodon, rulg, rad, rubra. Moris, H. reg. Bes. auct. p. 186.

Ornithogalum maritimum seu Scilla radice rubra. T'ouratefort. instit. rei herbar.

Ornithogalum maritimum Lamark Fl. franc. t. 3. p. $\$$ qb. Brot e ro flor. Lusitan. T. 1. p. 533.

Stellaris Scilla Manch methodus plant. p. 303. Ornithogalum Squilla Bat. mag. t. 794. Scilla maritima Linn. cum ampla Synonymia!

U. Horibus crebris dense racemosis albidis pedunculo brevioribus, pericarpiis angalatis latis, scapo tereti, bulbo maximo, foliis serotinis, latioribus obtusis.

Noch macht Herr S. auf die Fehlerhaftigheit vieler $\mathbf{\Delta b}$ bildungen der Meerzwiebel autmerksam, zumal was den Bau der Saamen angeht, der doch schon von Clusius ganz naturgetren gezeichnet worden sey.

Am interessantesten ist, was Herr S. über das Wachsthum der Zwiebel der Scilla maritima sagt: Sie ist aus Schuppen gebildet, die aufsen trocken sind, und um so fleischiger werden, je weiter man pach innen kommt; swischen ihnen findet man keine Spur von dem Schafte des vorigen Jahres, en dafs man annebmen mufs, die Schuppen erneuerten sich alle Jahre,

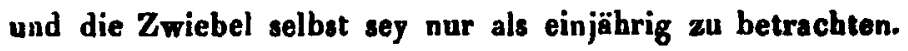

Selten findet man Augen in den Schuppenwinkela, und 
genau im Mittelpunkte der Z Wiebel entwickelt aich der Schatt, umwickelt von der letzten Schuppe! in dem Wiakel dieser nïmlichen Schuppe, zwischen ihr and dem Schafte, findet sich oin kleiner Trieb (bourgeon), ans dem die Bltitter hervorhommen, sie entwickeln sich maeh der Blame, and indem sie die alten Schuppen zarüchdrängen, legen sio den Grund zur Zwiebel des nächsten Jabres. Zu gloicher Zeit erocheinen Wurzelfasern, die kronenformig über jener Schoibe (platean) sich bilden, die ron den Ueberbleibseln des Stengels und der alten Wurzeln erzengt wird, die nach unten und innen gedrücht werden and allmählig rerschwinden; os exiatirt also bei der Reproduction der Theile eine doppelte Berrogung, die der Blätter oder Schuppen, die ron unten nacb oben und ron innen nach aufsen Statt hat, und die der Wurzelfasern, die von oben nach unten und ron aufsen nach innen geschieht. Da diese beiden Thätigheiten in entgegengesetztem Siane ror sich gehen, so neatralisirea sio sich wechselseitig, und das Resultat ist, dafs die Zwiebel immer on der nämlichen Stelle bleiben mufs. Die Entwickeinng dor Blätter erreicht ihre böchste Ausbilldung einige Zeit nach der Reife der Früchte, nämlich zo Ende des Winters, and diesos dauert fort darch die Bildung innerer Schuppen bis zum September, sie ist beendet durch das Entstehen eines Blamen. tragenden Schaftes, währenddem ein Kleiner Seitentrieb, in dem Winhel der innersten 6chuppe, dieselbe Erolution roa Neuem beginnt. Die Stellung der Theile zeigt offenbar, dafs der Schaft endständig (terminal) ist uod somit sich aut dieselbe Art ealwickelt, wie die Blätter, während der Whaine. Trieb nur seitenständig (lateral) ist, weil er zwischen dem Schafte und der Mittelrippe der letzten Schappe liegt. (Annales des Sciences naturelles. Seconde série Vol. 1. p. 321 u. d. f.) 


\section{Sarsaparilla.}

Nach Herrn Bichard Battley unterscheidet man auf dem Londoner Morkte die Sarsaparill ron Lissabon, Honduras, Vera.Cruz, Jamaika und Lima, auch hat man neuerlich aus Bombay und vor noch kürzerer Zeit aus Sierra Leona welcbe eingeführt. Diese Arten Lommen von nicht weaiger als fünf verschiedenen Pflanzen. Die afrikanische Sarsaparille unterscheidet sich sebr von den übrigen Sorten, und besteht aus rollknmmen glatten, schlaffen Fasern, deren braune federartige Rinde den schwachen holzigen Hern lose amgibt, und die aus einem festen Stocke (chump) entspringen. Die Lissaboner kommt aus Brasilien, bat ein glattes schmutzigbreunes Häutchen, und enthält in der Rinde, so wie im Kerno viel Stärke. Die einander sehr ähnlichen Sorten von Vera. Craz, Lima und Jamaika haben eine runzliche Obertläche, eine dünne, leicht ebzntrenuende Rinde, kaum einen Kern, und um das Holz heinen mehlartigen Stoft, wie das Lissaboner. Jedoch hat die von Jamaika eine röthliche Oberhaut und ist häufig mit kleinen Fasern bedeckt, während die von Vera-Cruz und Lima dūnner, mit solchen Anhängseln sparsamer besetzt, und letztere häufig mit dem Wurzelstocke versehen ist. Die Jamaika.Sarsaparille Hommt eigentlich ron dem Theile der Hondurashüste, welcher Mashito-Küste genannt wird. Die Honduras-Sarsaparille bat eine runaliche Oberfläche, dicke Rinde und Mark, welche viel Stärke ento hält. Die ostindische kommt zu uns in kurzen Stücken, von verschiedener Dicke, die sebr runzlich sind, ein aufgesprungenes, rauhes, der Ipecacuanha nicht unähnliches Ansehen baben. Die Rinde ist braun, dick, hart, apröde, geht beim Stofsen im Mörser leicht $a b_{;}$worauf ein holziger zöher Hern ohne Mark übrig bleibt. Sie scheint ganz rerschieden zu seyo roa der in Ainslie's Materia medica Vobl. 1. p. 38ı. beschrie. 
benen Wurzel der Periploca indica oder "Country-Sarsa. parilla a.

In den folgenden Tabellen suchte man eine Vergleichung zu geben, wieviel Extract diese verschiedenen Sorten Sarsaparille liefern, wieviel sie unzerstörbare Bestandtheile entbalten, and endlich, wie sich in dieser Hinsicht die ganzen Wurzeln and ibre Theile, das Holz und die Rinde zu einander verhalten. Die zu jeder Analyse verwendete Quantität betrog zwei Dnzen sorgfältig gereinigte Wurzel, die man zuerst mit kaltem, dann mit warmem Wasser macerirte. Beide Aufgüsse waren stets sauer and enthielten leine Stärke. Dana wurde die Masse gekocht und der Bückstand getrocknet. Die Ablochung war nicht sauer, enthielt aber Stärke. Die Aufgüase und die $\Delta$ blochung warden einzeln za trockenen Extracten abgeraucht, und diese nebst dem Rückstande zuerst in einem Wedgewood-Tiegel eingeäschert. Die Asche von jedem warde mit destillirtem Wasser gehocht und die Auflosungen mit salpetersaurem Silber auf Salzsäare, mit salpetersaurem Baryt anf Schwefelsäure geprüft. Aus den sodann concentrirten Auflösangen wurde gewöhnlich durch Weinsaure Hali abgeschieden. Der in Wasser unauflösliche Theil der Asche wurde hierauf in verdünnter Salpetersäure aufgelöst and mit Eisencyankalium auf Eisen, mit Ammoniak auf Thonerde, und mit Hoblensaurem Natron auf Halk gegeprüft. - Es lieferten anf diese Weise an Extract (nach Granen berechnet):

\section{I'on der Sarsaparille von Lima:}

- Unzen Wurzein: 87 Gr. Extract durch kalten Aufgufs, $45 \mathrm{Gr}$. Extr. durch warmen Aufgufs, $16 \mathrm{Gr}$. Extr. durch Ablochung und 68,5 Rückstand. 2 Unzen Rinde: 126 durch balten Aofgufs, 44 darch warmen Aulgufs, 38 durch AbHochung und 564 Rückstand. 2 Onzen Holz: 122 darch lalten 
Aufgufs, 34 durch warmen Aufgufs und Ablochung und 756 Rückstand.

II. Von der Sarsaparille von Jamaika:

2 Unzon Wurzeln: 232 Gr. Extr. and 540 Gr. Rückstand.

2 Rinde: 282 $\downarrow \geqslant 553, *$

2 . Holz: 100 " $>725$,

III. Von der ostindischen Sarsaparille:

2 Onzen Wurzeln: 150 Gr. Extr. durch balten Aufguf, $7^{3}$ Gr. Extr. durch Ablochung and 673 Rückstand.

1V. Van der Lissabuner Sarsaparille:

2 Unzen Wurzeln: go Gr. Ertr. durch halien Aufguls, 24 Gr. Extr. durch warmen Aufgufs, 102 Gr. durch Abkochung und 6,6 Rückstand.

V. Von der afrikanischen Sursaparille:

a Unzen Fasern: 26 durch Aufgurs, 25,3 durch $\mathrm{Ab}$ bochung und 580 Rückstand; 3 Unzen Wurzelstock: 77 durch Aufgufs, 22 durch Ablochung und 694 Rückstand.

Die Jamaika-Sarsaparille hat sich neuerdings sehr verschlechtert, da im Jahr 1822 ron einem Pfund 6 Unzen Extract, gegenwärtig selten mehr als 4 Unzen erhalten worden. Uebrigens erbielt man, als 7 Unzen Wurzeln derselben Sorte mit kaltem Wasser macerirt wurden, aus diesem Aufguis durch Hinzusetzung einer concentrirten Auflösung von Weinsäure einen Haufen Krystalle, die, nachdem sie mit rectifcirtem Weingeist abgewaschen waren, 42 Gran wogen und aus doppelt-weinsanrem Hali bestanden. Dieser Weingeist war dudurch gebräunt worden und hatte etwas Harz anfgenommen, dach wird ron pulverisirter Sarsaparille sowobl balter als Hochender Weingeist haum gefärbt. Halter Aether von spec. Gew. $=0,733$ bildete eine blafsgelbe Auflösung, die mach Verdampfung etwas Wachs zurüchliefs 2 Unzen Lissaboner 
Sarsaparillwurzel wurden in Wasser ron $110^{\circ}$ F. $\left(=35^{\circ}\right.$ R.) und dann ron $160^{\circ}$ F. $\left(=57^{\circ}\right.$ R.) macerirt, letzterer Aufgufs enthielt etwas Stärke; die hieranf vorgenommene vollständige Ablochung, welche kanm gefirbt war, lieferte darch Abranchen $200 \mathrm{Gr}$. trockene Substanz, die fast ganz aus Stärke bestand. Während der Maceration in heifsem $\mathbf{W}$ asser and besonders während des Kochens gibt die Sarsaparille einen sturken Gerach, den man anch in dem ans dem baiten Aúf. gufs bereiteten Destillate wahrnimmt. Auch bat der Perf. aus den Sorten roo Bombay und Jamaika ein wesentliches Oel erbalten, ron dem jener Geruch abhängt. Die Sareaperille scheint demnach Schwefelsänre, Salzsänre, Kali, Eisen, Thonerde, Kalt, Stärle, Harz, Wachs und ein wesentlicbes Oel za enthalten, wozu noch Gummi, Hleber, Farbeatoff and etwas Kieselerde hinzuhommt. Eine zweimalige Maceration von 2 Onzen Jamaika-Barsaparille in Wasser ron $35^{\circ} \mathbf{R}$. lie. ferte 110 Gr. trockenes Extract, während man durch $A$ b. kochung einer gleichen Menge $128 \mathrm{Gr}$. erhielt, wobei etwas Stärke war. Wegen des reicheren Gehaltes an Stärhe gaben daher auch einige Sorten durch die Ablochung mehr Extract, als die Wurzeln ron Lima. Da nun Präparate, welche Stärtse enthaken, der Verderbnifs leichter ausgesetzt sind, so ist ihre Darstelleng bei niedriger Temperatur vorzuziehen. Hierzu kommt noch, dafs durch Anwendung höberer Tomperetar wesentliches Oel verloren geht.

Man macerirte, um diefs zu ermittelo, I Pfund ron jeder Sorte in einem Destillirkolben und sammcite das hieraof destillirte Wasser, welohes den eigenthümilicben Sarsaparillengeruch besals und, wie die Berechnngg aach der opecifischen Schwere zeigte, an flüchtigem Stoffe bei der Jamailua-Sarsaparille go Gr., bei der Lissaboner $45 \mathrm{Gr}$, bei den übrigen gegen 3o Gr. enthiclt. Rechnet man diese Gewichte zn dem des darch gelindes Abrauchen orhaltenen and getrockneten 
Extractes, so ergibt sich immer noch ein Verlust ron 1) a Unz. 2 Dr. 34 Gr. bei der S. von Lima, 2) 2 Unz. 7 Dr. 8 Gr. bei der S. von Jamailia, 3) 2 Unz. 7 Dr. $33 \mathrm{Gr}$. bei der 8. von Lissabon, 4) 5 Unz. 4 Dr. $44 \mathrm{Gr}$. bei der S. von Bombay, 5) 5 Unz. 4 Dr. $49 \mathrm{Gr}$. bei der afrikanischen Sarsaparille. Der Haupiverlust ereignet sich daher, wenn die Masse ron der Consistenz des pharmaceutischen Extractes volleads zur Trockenheit gebracht wird. Die geeignetste Form zur Anwendung der Sarsaparille ist ohne $Z$ weifel das Pulver der Rinde oder der kalte Anfgufs der Rinde, den man auch, nachdem er 2 Tage lang ruhig gestanden und sich der gummige Stoff abgeschieden hat, zu einem Liquor concentriren bann. (Aus der Lond. med. Gazette Vol. I. Jan. 4. 1834. v. Schmidt's Jabrbücher Bd. 4. Heft 2. S. 2. u. d. f.)

Herr T. Martens, Provisor in Leipzig, schrieb Beitrăge zur medicinischen und pharmaceutischen Geschichte der Sarsaparilla; nach ihm ist S. Honduras, sowohl nach ihren chemischea Bestandtheilen, als nach therapeutischen Beobachtungen die vorzüglichste Sorte, wie schon $\mathrm{Hancoch}$ angab; defsgleichen lieferte das Extractum spirituoso-2quosum der Honduras eine recht passende Zubereitung. Diese Wurzel

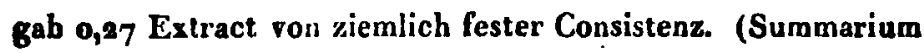
des Neuesten Bd. 1. Heft 8. 1836; Schmidt's Jahrb. Bd. I1. Heft 2. S. 155.)

C. Gowächse aus der Abtheilung der Zellenpflanzen
oder Akotyledonen.

Es sind deren bekanutlich im Ganzen nur wenige hent 20 Tage officinell und gebräuchlich, doeb benutzte man in den letzteo Jahren mehrere Algen als diätetische und Heilmittal, theils als Nutrientia wegen ihres reichen Gehaltes an indifferenten Stoffen, theils als Dissipantia und Resolventia, da mebrere dieser Meerbewohner iodhaltig sind und somit in 
der That wirksume Arzneimittel abgeben bơnnen. Es aind für jotzt nur folgende Tange zu erwähnea.

\section{Sphaerococcus mammillosus A ghard et Lyng by.}

Ein Tang, der zumal in der Nordsee and dem atlantischen Meere häufig gefunden wird; Decandolle sah ihn blos als eine Varietät der Ulva crispa an; La mouroux nennt ibn Fucus polymorphus, quarta series; anch anter den Namen Fucus ceranoides, Fucus spermophorus, F. canaliculatus, F. alveolatus a. s. w. hommt er in den Schriften der Botaniler vor. $\triangle$ ghard gibt folgende Definition:

S. mammillosus: fronde subcanaliculata dichotoma, segmentis lineari-cuneiformibus, capsulis sphaericis in disco frondis sparsis brevissime pedunculatis.

Nach Herrn Professor Henschel in Breslau constituirt diese Alge zugleich mit dem Sphaerococcus crispas und vielen Varietäten beider Arten das jetzt schon ziemlich verbreitete Carragaheen der Apotheken. (Uebersicht der Arbeiten and Vorhandlungen der schlesischen Gesellachaft etc. Breslau 1835 8. 103.)

Sphaerococcus acicularis A g hard.

Fronde cartilaginea filiformi subdichotoma, ramis patentibus acuminatis, ramenta lateralia horizortalia spiniformia ferentibus, capsulis sphaericis sessilibus sparsis.

Fucus acicularis Wulfen,

Fucus spinosus Gouan,

Fucus plicatus Clem.

Gigartina acicularis Lamouroux.

Man findet diese Alge im atlantischen Meere, von den Hüsten des nördlichen Franlireichs an, bis nach Afrika; auch Anaal. d. Pharm. XX. Bds. 3. Heft. 
306 Botanische und pharmakognostische Neuighellem.

im mittelläodiecthen und adriatisehen Meere kommt sie rot. Sie ist purpurröthlich and wird getrocknet noch dunkler, ibre Consistenz ist knorpelartig und getrocknet wird sie ganz steif. Lange macerirt nimmt sie eine rosenrothe Farbe an and geht endlich vollständig in eine Gallerte über. Bisweilen, setzt $\Delta g$ hard hinza, verwandelt sich die röthliche Farbe dieses Gewächses in eine grünspaaartige.

Nach Dro Giovanni Domenico Nardo in Venedig liefort diese Seeplanze eine vorzügliche Gallerte, die er als Heilmittel gegen verschiedene Lungenkrantheiten rübmat. (Botan. Zeitung 1835. Bd. 1. S. 38.)

\section{Sphaerococcus cartilagineus Aghard.}

Fronde cartilaginea filiformi compressa decomposito - pinnata, pinnis horizontalibus alternis, pinsulis apice capsuliferis, capsulis ellipticis mucronatis.

Linné nannte diese Alge Fucus cartilagineas, in Gmelin's Sebriften kommt sie als Fucus capensis und veroicolor vor, Lamark nannte sie Gelidium versicolor und Gelidium concatenatum etc. Es gibt davon eine Varietät mit borstenförmigen Verzweigungen (var. setacea) und findet sich am Cap der guten Hoffnung, so wie im indischen Meere.

Nach A ghard ist die gedachte Abart jene Alge, womit die Schwalben ihre Nester bauen, und die auch in den indischen Apotheken unter dem Namen Corallina japonica rerhauft wird.

Herr Prof. Meyen in Berlin mochte in den jüngsten Zeiten darauf aufmerksam, dafs jene varietas setacea in Japan zur Heilung des Hropfes benutzt wird. Allem Ansehen nach rerdankt sie diese Wirkungsart ihrem lodgehalte. 


\section{Pharmakognostisches Curiosum.}

Wegen Verwechselung ron Erysimum, welches ron einem Ehepaare in Paris gegen Catarrh im Aufguls gebreucht werden sollte, mit Blättern von Datura Strumonium, welche sich oin Apotheker.Lehrling hatte zu Schulden tommea lassen, und wo dann der Genufs des Infusums Vergiftungszufälle reranlafste, die aber durch einen dazugerufenen Arzt gehoben wurden, ist der Apotheler zu 100 Francs Strafe and 600 Francs Entschädigung für die Vergiftetgewesenen und aufserdem noch in die Kosten verurtheilt worden. (Berliner medic. Central. zoitung 1836. S. 664.)

Mas dart wohl mit Grend hoffen, dafs 20 etwas heat 24 Tage in Deatschland nicht mehr-vorkommen wird, denn die Verwechselung einer planta crucifera mit einer planta solanacea rerräth 20 grobe Unwinsenbeit in den Anfangsgründen der Botanik, dafs sie selbst bei einem Lehelinge unrerzeih. lich ist.

Neue Nachrichten von den schädlichen Eigenschaften der Pflanzen.

Nachtheilige Wirkung des Wurmsaamens.

Hreisphysikus Dr. Hoffmann in Suhl beobachtete zwelwal bei Kindern, nach Darreichung des Semen Santonici, einmal mit etwas Rheum, das anderemal ganz allein, sehr heftige Hirnaffectionen. In einem Falle, bei einem siebenjährigen, eben nieht schwäehliehen Hnaben, mufste $H$. sogar etaige Blutegel an die Schlïfe setzen lassen und kalte Omschlïge verordnen, worauf der Hnabe nach 5 Tagen hergestelt war. 
Daf hier zufällige Anfeinanderfolge der Umstïnde anzanohmen sey, glaubt $H$. defshalb nicht, weil er wohl bei 12 Kin. dern nach Wormsaamen allemal dasselbe und ohne andere nachweisliche Ursache erfahren hat. - Diese Beobachtung ist auch nicht neu, sondern es haben schon mehrere, and neuerdings noch Jahn, ror solchen methodischen Wurmkuren, die der gemeine Mann gern jedesmal im März bei abnehmendem Monde vornimmt, gewarnt. Worauf übrigens die grofse Reizbarbeit gegen dieses Mittel begründet sey, ob anf eigenthümlicher Wirkung des ätherischen Oels des Sanmens der Artemisia Contra (? D.) oder anf gesteigerter ner-

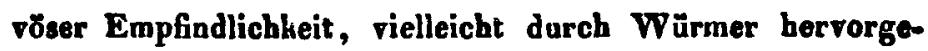
bracht, wagt $H$. nicht zu entscheiden; arch kann er lueine Vorsichtsmafsregeln hinsichtlich der Auswahl der Subjecte für solche Kuren rorsoblagen, da sebr verschiedenartige Naturen ron diesen Zufällen befallen wurden. Vom Extractum Cinae setheroum nahm H. etwas Aebnliches noch nicht wehr, doch wendete er auch dasselbe, weil es theuer ist, nur selten an. (Medic. Zeitung rom Vereiue f. Heilkunde in Preufsen 1836, No. 34 ; Scbmidt's Jahrb. Bd. 11. Heft 2. 8. 163.)

Schädliche Wirkung von blühendem Flachs (Linum usitatissimun) als Viehfutter.

Schon ofters ist in offentlichen Blättern die schädliche Wirtung erwähnt worden, welche blühender Lein anf das damit gefütterte Vieh äufserte, indem er in einzelnen Füllen Vergiftungszufälle und selbst den T'od der Thiere wach sich gezogen baben soll. Ein Fall der Art hat sich lüralich zu Spöb bei Karlsrube zugetragen. - Es sah sich nämlich ein dortiger Güterbesitzer veranlafst, einen in Blüthe gestaodenen Lein, seines schlechten Bestandes halber, auszurupfen, um ihn 
nachber als Streu zu benutzen. Die Küho, welche zum Einfabren dieses Leines gebraucht warden, frafsen daron, und awar haum 8 Minuten lang, so dafe an ein Ueberfressen oder eine Anfblähung nicht zu deuken war; dennoch trat die Wir kang so schnell ein, dafs die eine der Hühe gleich darsuf todt binstürzte and die andere gleiches Schicksal gefunden hätte, wenn sie vicht zeitig genug durch Eingüsse von Easig and Leinsamen "), 20 wio durch wiederholte Hlystiere gerettet worden wäre. Bei der Section des gefallenen Thieres war blos wabrzanehmen, dafe sich die Futtermasee in der dritten Abtheilang des Magens, dem Psalter (Blättermagon, Mannigfalt), ganz feat zasammengedrängt hatte. (Grofsh. Bad. land. wirthsech. Wochenblatt 1836. S. 146.)

\section{Vergiftungszufall durch wilden Mohn,}

Papaver Rhoeas L.

Der Bericht über diesen Fall rührt von dem Thierarzto $8 \mathrm{chmager}$ in Lahr her, er ereignete sich in Dinglingen mit zwei Kühen, die plötalich von einer der Epilepsie ähnlichon Krankheit befallen warden, die Thiere zitterten, bekamen Schaum vor den Mand, waren einige Zoit bewafstlos and hatten heine Empfindungen mehr, man bemertte ferner convalsivische Bewogungen und Verdrehungen der Augen, so wie der Extremitäten, des Halses und Hopfes. Von Zoit zu Zeit schienen die Thiere ruhig zn werden and zo schlafen, allein die Anfälle wiederholten sich oftere unter beftigem Basen und Toben, das biء zu völliger Erschöpfang der Kräfte fortdauerte. Das antersuchte Futter bestand, aufser einigen Gras.

7) Auffallend ist es, dafs hier der Saame des Linum usitationimum als Antidestum der blühenden Planze gereicht wurde: 
arted, fast durohgeheade aus Papaver Bhoees, der schon gana abgeblübt war, und eine Menge unreifer Saamenkapoela exthielt, aus welchen beim Drücken viel milchiger ulebriger Saft ausffofs, und wobl ganz richtig verwuthet Herr 6 ., dafs dieser Milcheaft dieselben hehannten Bestandtheile besitze, wie der vou Papaver somniferum, aus welchem das Opiun bo. reitet wird. Die Zafälle der Thiere betrachtet man demnach als Symptome einer narhotischen Vergiftung und reichte ihnen eine Mischung aus roinem Weinosig mit Oel, und abwechselnd echwarzen Haffee, wodurch denn anch die Hauptzufalle binnen 2 wolf Sto.uden beseitigt wurden, und nach 24 Standen hörten sie günalioh auf; die Thiere fingen dann wieder all. mählig an zu fressen, und man bcmerlte aufser einem düstern Blicke und Schiefstehen, nichts Kranthaftes mehr an ihnen. (Daselbst 1836. S. 879.) 